\title{
Probing nuclear modifications of parton distribution functions through the isolated prompt photon production at energies available at the CERN Large Hadron Collider
}

\author{
Muhammad Goharipour ${ }^{1, *}$ and S. Rostami ${ }^{2, \dagger}$ \\ ${ }^{1}$ School of Particles and Accelerators, Institute for Research in Fundamental Sciences (IPM), P.O. Box 19568-36681, Tehran, Iran \\ ${ }^{2}$ Young Researchers and Elites Club, South Tehran Branch, Islamic Azad University, Tehran, Iran
}

(Received 19 September 2018; revised manuscript received 25 December 2018; published 20 May 2019)

\begin{abstract}
An accurate knowledge of nuclear parton distribution functions (nPDFs) is an essential ingredient of high energy physics calculations when the processes involve nuclei in the initial state. It is well known now that the prompt photon production in both hadronic and nuclear collisions is a powerful tool for exploring the parton densities in the nucleon and nuclei, especially of the gluon. In this work, we are going to perform a comprehensive study of the isolated prompt photon production in $p$ - $\mathrm{Pb}$ collisions at backward rapidities to find the best kinematic regions in which the experimental measurements have most sensitivity to the nuclear modifications of parton densities. Most emphasis will be placed on the antishadowing nuclear modification. To this aim, we calculate and compare various quantities at different values of center-of-mass energy covered by the CERN Large Hadron Collider and also different rapidity regions to realize which one is most useful.
\end{abstract}

DOI: 10.1103/PhysRevC.99.055206

\section{INTRODUCTION}

Besides the parton distribution functions (PDFs), whether unpolarized [1-11] or polarized [12-18], and fragmentation functions (FFs) [19-23], the nuclear modifications of PDFs [24-31] are also important ingredients of high energy physics calculations, in particular for processes involving nuclei in the initial state. In fact, without having nuclear PDFs (nPDFs) which describe the structure of the colliding nuclei, the theoretical calculation of the cross sections in any nuclear collision will not be possible. Thanks to the collinear factorization theorem $[32,33]$, the nPDFs can be extracted in a way similar to the PDF determination through a global analysis of nuclear experimental data. Nowadays, due to many developments achieved in the phenomenological approaches, theoretical calculations, and experimental measurements, the PDFs are well determined in a wide range of the momentum fraction $x$. However, the situation is not very satisfying for the case of nPDFs because of the lack of experimental data.

Although the main experimental data for constraining nPDFs come from the old fixed-target deep inelastic scattering (DIS) and proton-nucleus Drell-Yan (DY) dilepton production experiments, there are some analyses in which the neutrino DIS data have also been used $[25,27,30]$. Furthermore, the inclusive pion production from $d$-Au collisions at the

\footnotetext{
*muhammad.goharipour@ipm.ir

†asalrostami.phy@gmail.com
}

Published by the American Physical Society under the terms of the Creative Commons Attribution 4.0 International license. Further distribution of this work must maintain attribution to the author $(\mathrm{s})$ and the published article's title, journal citation, and DOI. Funded by $S C O A P^{3}$.
BNL Relativistic Heavy Iion Collider (RHIC), that can be considered as another source to put further constraints on the nuclear gluon distribution, is usually used in the nPDF analyses [26-28,30]. Recently, the nPDF set "EPPS16" [30] also included, for the first time, the fixed-target DY data in pion-nucleus collisions and new CERN Large Hadron Collider (LHC) proton-lead ( $p$ - $\mathrm{Pb})$ data on dijet and heavy gauge-boson production. There are also some studies showing that important information about nPDFs can be achieved by analyzing the prompt photon production in nuclear collisions [34-39], jet and dijet photoproduction measurements at a future electron-ion collider (EIC) [40,41], single inclusive jet production at very forward rapidity [42], and heavy-flavor production in $p-\mathrm{Pb}$ collisions [43].

The main reason for such efforts to include more accurate experimental data as much as possible in the global analysis of $\mathrm{nPDFs}$ is to achieve more valid nuclear modifications of PDFs with less uncertainties. In fact, most of experimental data that are used in the nPDFs analyses can put good constraints on the quark nuclear modifications at fairly large values of $x$, while the sea quarks and gluon distributions cannot be controlled as well, especially at smaller values of $x$. Actually, the limited kinematic reach of data leads to a crucial difference between the results obtained by various groups for the gluon nuclear modifications and also their uncertainties. Therefore, since the common constraint of nuclear gluon distribution that comes from the Dokshitzer-Gribov-Lipatov-AltarelliParisi (DGLAP) evolution at higher order of perturbation theory is not enough to make accurate theoretical predictions of the physical observables, the inclusion of experimental data that are directly sensitive to the gluon density in the nPDFs analysis is inevitable.

It is well known now that the direct photon production in hadronic collisions is one of the excellent tools for studying 
the large momentum transfer processes [44-48] and thereby testing perturbative quantum chromodynamics (pQCD), since photons couple in a point-ike fashion to the quark constituents of the colliding hadrons. Direct photon production is also recognized as a useful tool [49] for studying the whole time evolution and dynamics of the deconfined, strongly interacting matter, namely the quark gluon plasma (QGP) [50,51], created in heavy-ion collisions. It is worth noting in this context that in nucleus-nucleus collisions, direct photon production contains two kinds of photons: thermal photons and prompt photons [52]. The first ones have a thermal origin and the second ones come from cold processes. By definition, the prompt photons do not include the photons coming from the decays of hadrons such as $\pi_{0}, \eta$ produced in the collision. The measurement of the prompt photon production in hadronic collisions can bring very useful information of gluon PDFs [53-57] and, as mentioned before, the gluon nuclear modifications [34-39] in the case of nuclear collisions. Such measurements have other advantages in various areas of high energy physics, for example searching for the intrinsic heavy quark components of the nucleon if photons are associated with a heavy quark [58,59]. Another important point that should be mentioned is that, in order to reject the background of photons that are not considered prompt photons, an isolation criterion is usually used.

In view of the experimental efforts, there are various measurements of direct photon production in heavy-ion collisions by different collaborations. For example, on can refer to the PHENIX Collaboration measurements in Au-Au [60-63] and $d$-Au [64] collisions at RHIC and center-of-mass energy of $\sqrt{s}=200 \mathrm{GeV}$, and also the ALICE Collaboration measurements $[65,66]$ at the LHC from $\mathrm{Pb}-\mathrm{Pb}$ collisions at $\sqrt{s}=$ $2.76 \mathrm{TeV}$. For the case of isolated prompt photon production in $\mathrm{Pb}-\mathrm{Pb}$ collisions, there are also some measurements by the ATLAS [67] and CMS [68] Collaborations at $\sqrt{s}=$ 2.76 TeV. Despite all these experimental efforts, another kind of measurement that is very important for achieving accurate information of the gluon nuclear modifications is the measurement of the prompt photon production in $p-A$ collisions, especially at higher values of the center-of-mass energy and forward rapidities [39]. Recently, the ALICE Collaboration has reported the measurement of inclusive production cross section for isolated prompt photons in $p$-Pb collisions at $\sqrt{s}=$ $8.16 \mathrm{TeV}$ as a function of photon transverse energy from 20 to $550 \mathrm{GeV}$ and over three regions of pseudorapidity [69].

In this work, we are going to perform a comprehensive study of the isolated prompt photon production in $p$ - $\mathrm{Pb}$ collisions at different values of center-of-mass energy covered by the LHC and also different rapidity regions. Since the study of the shadowing nuclear modification of parton densities through the prompt photon production at the LHC has been done previously in some papers, in the present study more emphasis will be placed on the antishadowing nuclear modification. In fact, the main goal of this work is finding the best kinematic regions in which the measurement of the isolated prompt photon production in $p$ - $\mathrm{Pb}$ collisions has most sensitivity to the antishadowing area and so is more useful to constrain the antishadowing nuclear modification of the gluon distribution.
The contents of the present paper are as follows. In Sec. II, we discuss the nuclear modifications of PDFs with emphasis on gluon nuclear modification for the $\mathrm{Pb}$ nucleus and compare predictions of various phenomenological groups at factorization scales corresponding to energies covered by the LHC. Our framework and anything needed to calculate the isolated prompt photon production cross sections and other related quantities are briefly described in Sec. III. Section IV is devoted to studying the isolated prompt photon production in $p$-Pb collision at different values of center-of-mass energy and different rapidity regions to find the best kinematic regions for constraining the antishadowing nuclear modification of the gluon PDF. In this regard, various quantities, including the nuclear modification ratio, the rapidity and energy normalized nuclear modification ratios, and the ratio of nuclear cross sections for different rapidity regions and also different energies, are calculated and compared with each other to realize which one is most useful. We also present a comparison between the predictions obtained from various nPDFs and study the uncertainties due to scale variations and nPDFs. Finally, we summarize our results and conclusions in Sec. V.

\section{THE NUCLEAR MODIFICATIONS OF PARTON DENSITIES}

As mentioned in the Introduction, in order to calculate any cross section of the particles production in high energy nuclear collisions, it is necessary to have accurate nPDFs which describe the structure of the colliding nuclei. The determination of the nuclear modifications of parton densities usually has a procedure similar to the case of PDFs of free nucleons; that is, performing a QCD global analysis of nuclear experimental data [24-31]. Regardless of this fact that the determination of nPDFs with a precision similar to PDFs is not possible at the present due to the lake of nuclear experimental data, the different phenomenological approaches used by various groups to extract the nuclear modifications of parton densities have led to some considerable differences in the results obtained, both in behavior and uncertainty, especially for the case of gluon and sea quark nuclear modifications. The differences between the nPDFs from various phenomenological groups, in turn, can lead to the different results for the theoretical predictions of the physical observables. Therefore, in inverse, the accurate measurements of some particle production cross sections in nuclear collisions can bring us new useful information to judge about the behavior of nuclear modifications in different kinematic regions and also decrease their uncertainties. The measurement of the prompt photon production is one of the most useful ones to obtain information about the gluon nuclear modifications [34-39], since the gluon directly contributes to it through the Compton scattering $q(\bar{q}) g \rightarrow \gamma q(\bar{q})$ subprocesses at the Born-level.

From past to present, several QCD global analyses of $n P D F s$ have been done, which are different in various aspects such as the amount and kind of the experimental data included, the perturbative order of the analysis, the parametrization of the nuclear modifications, the method for calculating uncertainties, and treatment of the heavy quark masses (for reviews see Refs. [39,70]). In most of the analyses performed 
so far, like the HKN07 [24], EPS09 [26], DSSZ [27], KA15 [29], and EPPS16 [30] analyses, the bound-proton PDFs have been defined in terms of nuclear modification factors $R_{i}^{A}$ that characterize the relation of the PDFs of a proton inside a nucleus with atomic number $A, f_{i}^{p / A}$, with respect to freeproton PDFs $f_{i}^{p}$ :

$$
R_{i}^{A}\left(x, Q^{2}\right) \equiv \frac{f_{i}^{p / A}\left(x, Q^{2}\right)}{f_{i}^{p}\left(x, Q^{2}\right)} .
$$

In this approach, for determining the nuclear modifications one needs to fix the free-proton PDFs from an analysis already performed on the nucleon experimental data [1-11]. However, there is another approach in which the nPDFs are parametrized directly as a function of $x$ at the starting scale $Q_{0}^{2}$ without any factorization into a nuclear modification factor and free-proton PDFs. In this approach, that has been used by the nCTEQ group [25,28], the $A$ dependence of nPDFs is introduced in the coefficients of their functional form.

A comparison between nuclear modifications of the gluon $\mathrm{PDF}$ in a $\mathrm{Pb}$ nucleus with their uncertainties from the nCTEQ15 [28] (blue solid), EPS09 [26] (red dashed), DSSZ [27] (green dashed-dotted) and HKN07 [24] (pink dotted) is shown in Fig. 1. The comparison has been made for two different scales, $Q^{2}=1000$ (top panel) and 10000 (bottom panel) $\mathrm{GeV}^{2}$, which are covered by LHC energies. As can clearly seen, almost in whole range of $x$, there are considerable differences between the results of various groups in both best fits and uncertainties. Overall, the nuclear modification of parton densities, in terms of $x$, is usually divided into four areas, namely, shadowing, antishadowing, European Muon Collaboration (EMC) effect, and Fermi motion [70]. The antishadowing area, that is the favorite case of the present study, is attributed to an enhancement in $R_{i}^{A}$ around $x \sim 0.1$. The decreasing areas before and after it are named shadowing and EMC effect. There is another increasing area at very large values of $x$ which is referred to as Fermi motion. For the case of nCTEQ15, we see some stronger shadowing, antishadowing, and EMC effect in gluon nuclear modification compared to other groups that makes it a favorite case to use for studying this subject. Note also that the nCTEQ15 prediction has wider uncertainty than other groups in all values of $x$. Focusing on the antishadowing area, one can see that the EPS09 has a similar treatment to nCTEQ15 but is somewhat softer. The DSSZ does not show any remarkable antishadowing nuclear modification in gluon density and, for the case of HKN07, it appears at some larger values of $x$ in analogy to the nCTEQ15 and EPS09. Accordingly, one can easily conclude that such differences can lead to different results for predictions of any process in nuclear collisions which is sensitive to antishadowing nuclear modification of the gluon density.

In the prompt photon production at the LHC, RHIC, or any hadron collider, various $x$ regions can be explored in the PDFs of target and projectile according to following approximate relation:

$$
x_{1,2} \approx \frac{2 p_{\mathrm{T}}}{\sqrt{s}} e^{ \pm \eta}
$$
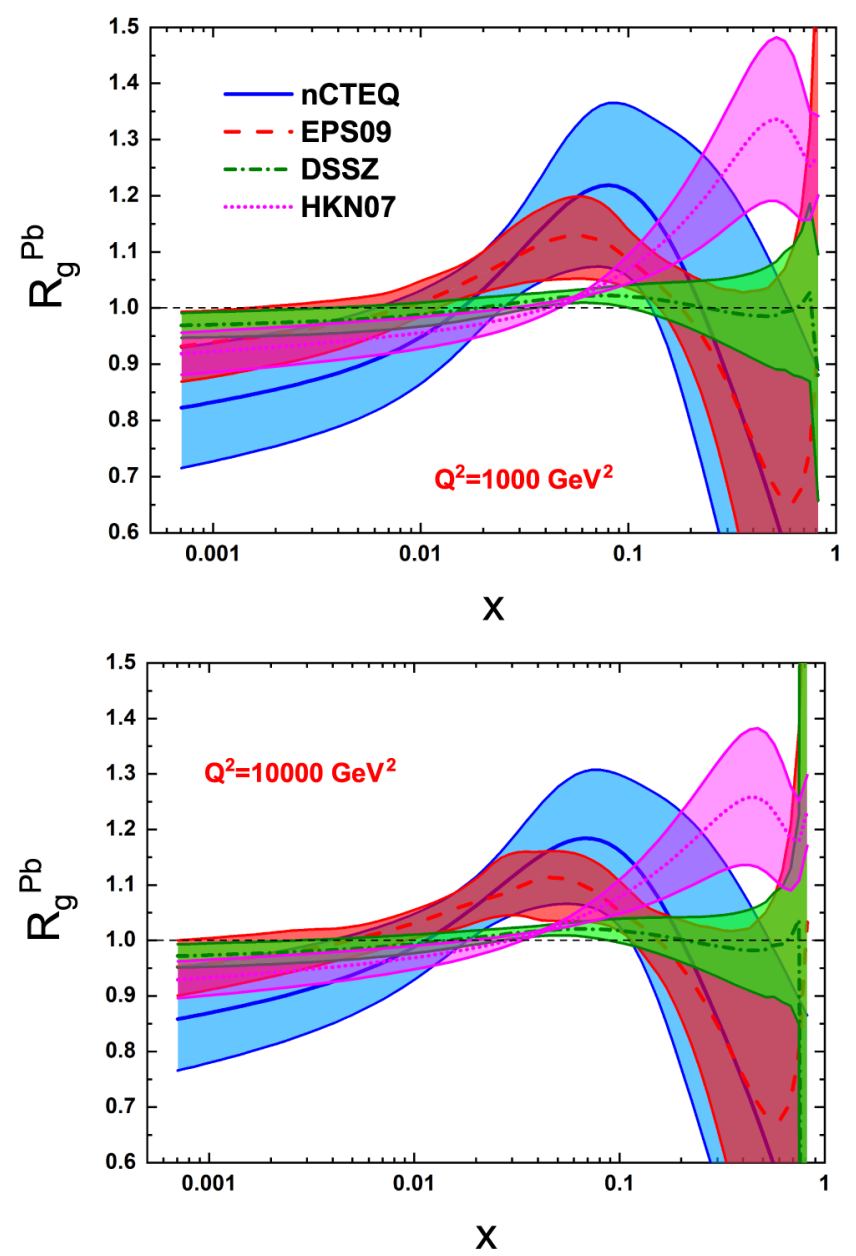

FIG. 1. A comparison between the nCTEQ15 [28] (blue solid), EPS09 [26] (red dashed), DSSZ [27] (green dashed-dotted) and HKN07 [24] (pink dotted) nuclear modifications of the gluon PDF in a $\mathrm{Pb}$-nucleus with their uncertainties at $Q^{2}=1000$ (top panel) and 10000 (bottom panel) $\mathrm{GeV}^{2}$.

which clearly depends on the center-of-mass energy $\sqrt{s}$ of the collision, and also the transverse momentum $p_{\mathrm{T}}$ and pseudorapidity $\eta$ of photons. According to Eq. (2), for the case of $p$-Pb collisions at LHC with high values of $\sqrt{s}$, we can only investigate the antishadowing nuclear modification at backward rapidities. Actually, at forward rapidities, the smaller values of $x_{2}$ can be probed, which are related to the shadowing area as studied before [34-39]. In this work, we are looking for the best kinematic regions in which the isolated prompt photon production at $\mathrm{LHC} p$ - $\mathrm{Pb}$ collisions has most sensitivity to the antishadowing nuclear modification and thus more potential to constrain it.

\section{THE FRAMEWORK OF THE CALCULATIONS}

As seen in the previous section, the gluon nuclear modifications extracted by various phenomenological groups have major differences almost in all regions of the momentum fraction $x$. This fact can lead to the different results for any physical observable which is dependent on the nPDFs. As 
mentioned before, in this work we are going to use the isolated prompt photon production in $p$ - $\mathrm{Pb}$ collisions at the $\mathrm{LHC}$ as a useful tool for investigating the gluon nuclear modifications, in order to find the best kinematic regions that have the most sensitivity to the antishadowing area. In this section, we briefly discuss the framework of the calculation of the cross sections.

The prompt photon production has been an interesting subject of research for more than three decades [34-39,44-48,53-57,71-88]. The prompt photons (those arising from processes during the collision, not those produced from the decay of hadrons) consist of two types of photons: direct and fragmentation photons. The conceptual difference between the direct and fragmentation photons is related to their production procedure. Actually, direct photons are produced directly from initial hard scattering processes of the colliding quarks or gluons, while fragmentation photons are produced from the fragmentation of high- $p_{\mathrm{T}}$ quarks and gluons which themselves were already produced during the hard collisions (for more information see, for example, Refs. [48,77]). The theoretical calculations of the direct and fragmentation components can be performed completely separately so that the prompt photon production cross section in hadronic collisions can be written as follows:

$$
d \sigma_{h_{1} h_{2}}^{\gamma+X}=d \sigma_{h_{1} h_{2}}^{\mathrm{D} \gamma+X}+d \sigma_{h_{1} h_{2}}^{\mathrm{F} \gamma+X},
$$

where D and F refer to the direct and fragmentation parts, respectively. Note that the notation $X$ is introduced to indicate the inclusive nature of the cross section. The cross section of the prompt photon production at hadronic collisions can be calculated through some computer codes such as JETPHOX $[48,77,78]$ and PETER [89] up to next-to-leading order (NLO). The next-to-next-to-leading order (NNLO) calculations of the direct photon production were also presented recently [88]. In the present study, we use JETPHOX to perform all calculations; it is a Monte Carlo program written as a partonic event generator for the prediction of processes with photons in the final state, and has also various features, for example using different procedures for isolating photons, as we discuss in the following.

Among the important points that should be considered in the calculation of the cross section of Eq. (3) are the values of the renormalization $\mu$, (initial state) factorization $M$, and fragmentation $M_{F}$ scales. The renormalization and factorization scales appear both in the direct and fragmentation components of the cross section, but the fragmentation scale appears only in the fragmentation part. Overall, due to scale variations, one can consider some uncertainties in theoretically calculated results (for more information about the scale uncertainties in the prompt photon production in hadronic collisions see, for example, Ref. [87]). In all calculations which are presented in the next section, the renormalization, factorization, and fragmentation scales are set to the photon transverse momentum $\mu=M=M_{F}=p_{\mathrm{T}}^{\gamma}$, except for the last subsection in which we study the uncertainties due to scale variations. To calculate the cross section of Eq. (3) one also needs to use the free-proton PDFs and FFs of the photons. Actually, the PDFs are used for calculating both the direct and fragmentation components, but the FFs are only used in the calculation of the fragmentation component. On the other hand, if we are interested to the proton-nucleus or nucleusnucleus collisions, we need also the nuclear modification of PDFs for each nucleus contributing in the collision. In this work, we use set II of the NLO Bourhis-Fontannaz-Guillet (BFG) FFs of photons [90] and the NLO PDF sets of CT14 [5] with $\alpha_{s}\left(M_{Z}\right)=0.118$ by virtue of the LHAPDF package [91]. For the nuclear modification of PDFs, we choose the nCTEQ15 nPDFs [28], that show stronger shadowing, antishadowing, and EMC-effect nuclear modifications according to Fig. 1. However, at the end of next section, we also present a comparison between the nCTEQ15, EPS09 [26], DSSZ [27], and HKN07 [24] predictions.

In hadronic collisions, different subprocesses contribute to the prompt photon production. In the case of LO approximation, they are the quark-gluon Compton scattering $q(\bar{q}) g \rightarrow$ $\gamma q(\bar{q})$ and quark-antiquark annihilation $q \bar{q} \rightarrow \gamma g$, both of which are Born-level subprocesses. At NLO, the situation is somewhat more complicated, since in addition to the $q(\bar{q}) g \rightarrow$ $\gamma g q(\bar{q})$ and $q \bar{q} \rightarrow \gamma g g$, there are some subprocesses from the virtual corrections to the Born-level processes. However, because of the pointlike coupling of the photon to quarks, such a calculation is easier than for other processes $[44,71,76]$. It is worth pointing out that at $p p$ colliders such as LHC and RHIC, the $q \bar{q}$ annihilation channel is suppressed compared to the other subprocesses. This is the main reason for the fact that the prompt photon production at the LHC provides direct information on the gluon distribution, especially at smaller values of $x$, because it is dominant rather than sea quarks in this region. The $q \bar{q}$ channel becomes more important at the Tevatron, a $p \bar{p}$ collider [55]. Another point that should be mentioned, regarding Eq. (2), is that the photon production at the LHC probes values of $x$ that are considerably smaller than at the Tevatron. In the present study, we include all diagrams up to LO and NLO order of QED and QCD coupling, respectively, defined in the modified minimal subtraction ( $\overline{\mathrm{MS}}$ ) renormalization scheme for calculating the prompt photon production cross section. Note also that the fine-structure constant $\left(\alpha_{\mathrm{EM}}\right)$ is set to $1 / 137$.

In the experimental view, if we are interested in measuring the prompt photon production inclusively, the background of secondary photons coming from the decays of hadrons such as $\pi_{0}, \eta$ should be well rejected, since they are not prompt photons by definition. The best idea for doing so is imposing an isolation criterion for photons. Although there are different isolation criteria that can be used to isolate photons [77,92-95], the most used is the cone criterion [77], which is implementable also at the partonic level. The cone isolation criterion considers a photon as an isolated photon if, inside a cone of radius $R$ which is defined as $\left(y-y_{\gamma}\right)^{2}+\left(\phi-\phi_{\gamma}\right)^{2} \leqslant$ $R^{2}$ in terms of rapidity $y$ and azimuthal angle $\phi$ around the photon direction, the amount of hadronic transverse energy $E_{\mathrm{T}}^{\text {had }}$ is smaller than some value $E_{\mathrm{T}}^{\max }$.

$$
E_{\mathrm{T}}^{\mathrm{had}} \leqslant E_{\mathrm{T}}^{\max }
$$

The value of $R$ is usually set to $R=0.4$ in the experimental analyses of isolated prompt photon production [68,69,96-99]. However, $E_{\mathrm{T}}^{\text {had }}$ is considered both as a fixed value or a fixed 
fraction of the transverse momentum of the photon $p_{\mathrm{T}}^{\gamma}$ and a function of $p_{\mathrm{T}}^{\gamma}$. It should be also noted that the photon isolation significantly reduces the fragmentation components of the prompt photon cross section, since the isolation cut discards the prompt photon events that have too much hadronic activity around the photon [77]. In all calculations which are presented in this paper, we use a tighter isolation cut, $E_{\mathrm{T}}^{\text {had }}<2$, with $R=0.4$.

Another point that should be considered is the theoretical uncertainty in the results with respect to the various sources, including the PDF, nPDF, scale, and FF uncertainties. For more information about the impact of these sources on the uncertainties of the prompt photon production cross section at the LHC, one can refer, for example, to Ref. [39]. It can be shown that the dominant source of theoretical uncertainty is that arising from the scale uncertainties, and the uncertainty due to free-proton PDF and FF uncertainties can be ignored compared to the nPDF uncertainties. Although our main goal is performing a comprehensive study of the isolated prompt photon production in $p$ - $\mathrm{Pb}$ collisions to find the best kinematic regions for constraining the antishadowing nuclear modifications of PDFs, we study in the next section the nPDF uncertainties using the nCTEQ15 [28] nPDFs, in addition to scale uncertainties.

It is well known now that the minimum bias nuclear modification ratio is a very useful quantity for studying the nuclear modifications of parton densities [37-39]. It is defined as the ratio of the cross section of collisions containing a nucleus to the cross section of pure proton-proton collisions. For the case of prompt photon production in $p-\mathrm{Pb}$ collisions we have

$$
R_{p \mathrm{~Pb}}^{\gamma} \equiv \frac{d \sigma / d p_{\mathrm{T}}(p+\mathrm{Pb} \rightarrow \gamma+\mathrm{X})}{208 \times d \sigma / d p_{\mathrm{T}}(p+p \rightarrow \gamma+\mathrm{X})}
$$

Such a quantity is clearly more sensitive to the nuclear modifications of PDFs. Moreover, many sources of theoretical and experimental uncertainty are canceled to a large extent in the calculation and measurement of nuclear modification ratio $R_{p \mathrm{~Pb}}^{\gamma}$. On the theoretical side, we can refer to the PDF, FF, and scales uncertainties, since they are in both numerator and denominator. Actually, the main source of theoretical uncertainty in $R_{p \mathrm{~Pb}}^{\gamma}$ comes from the nPDF uncertainties. Another point should be considered is that $R_{p \mathrm{~Pb}}^{\gamma}$ is not normalized to 1 when no nuclear modifications in the parton densities are assumed because of the isospin effect. In the next section, we begin our study by calculating the nuclear modification ratio for isolated prompt photon production in $p$ - $\mathrm{Pb}$ collisions at different values of center-of-mass energy. Then, we introduce and calculate other possible quantities which can be useful for investigating the nuclear modifications of PDFs.

\section{STUDY OF ISOLATED PROMPT PHOTON PRODUCTION AT THE LHC}

After introducing the framework of the calculations, we are now in position to calculate the isolated prompt photon production in $p$ - $\mathrm{Pb}$ collisions in order to investigate the nuclear modifications of parton densities, especially of the gluon. As mentioned before, we are looking for the best kinematic

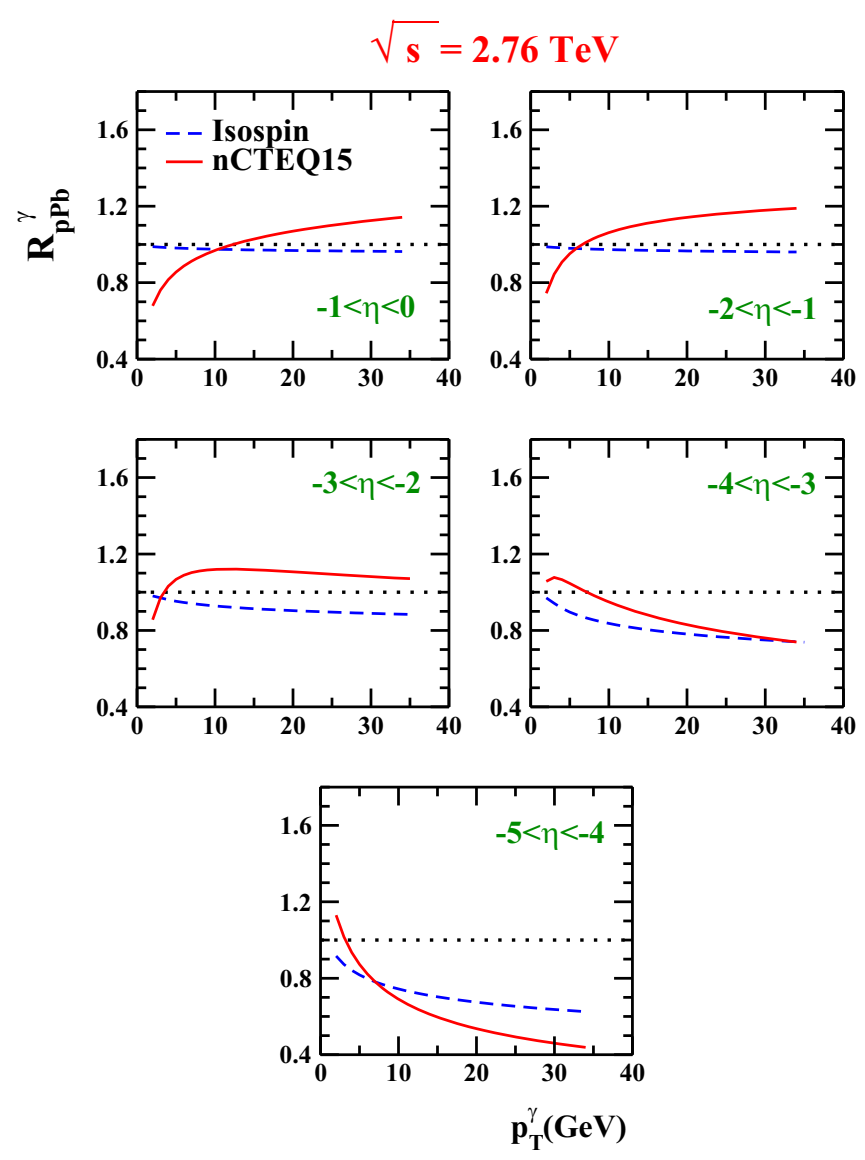

FIG. 2. The nuclear modification ratio $R_{p \mathrm{~Pb}}^{\gamma}$ as a function of $p_{\mathrm{T}}^{\gamma}$ for $p$ - $\mathrm{Pb}$ collisions at $\sqrt{s}=2.76 \mathrm{TeV}$ and five different rapidity regions in the backward interval $-5<\eta^{\gamma}<0$, with no nuclear modifications in the PDFs (blue dashed curve) and using the nCTEQ15 [28] nuclear modifications (red solid curve).

regions in which the measurement of the isolated prompt photon production in $p$ - $\mathrm{Pb}$ collisions has most sensitivity to the antishadowing area. To this aim, in the following subsections, we calculate and compare various quantities, including the nuclear modification ratio, the rapidity and energy normalized nuclear modification ratios, and the ratio of nuclear cross sections for different rapidity regions and also different energies to realize which one is most useful.

\section{A. Nuclear modification ratio at backward rapidities}

As a first step, we calculate the nuclear modification ratio $R_{p \mathrm{~Pb}}^{\gamma}$ defined in Eq. (5) for $p$-Pb collisions at $\sqrt{s}=2.76 \mathrm{TeV}$. As mentioned before, at such energies and higher ones, it is not possible to investigate the antishadowing nuclear modification at forward rapidities since, according to Eq. (2), the cross section is sensitive to parton densities of the target at smaller momentum fraction $x_{2}$.

Figure 2 shows the results obtained as a function of photon transverse momentum $p_{\mathrm{T}}^{\gamma}$ at five different (pseudo)rapidity regions in the backward interval $-5<\eta^{\gamma}<0$. In each panel, we show both the calculation with no nuclear modifications in the PDFs, which indicates only the isospin effect (blue dashed 


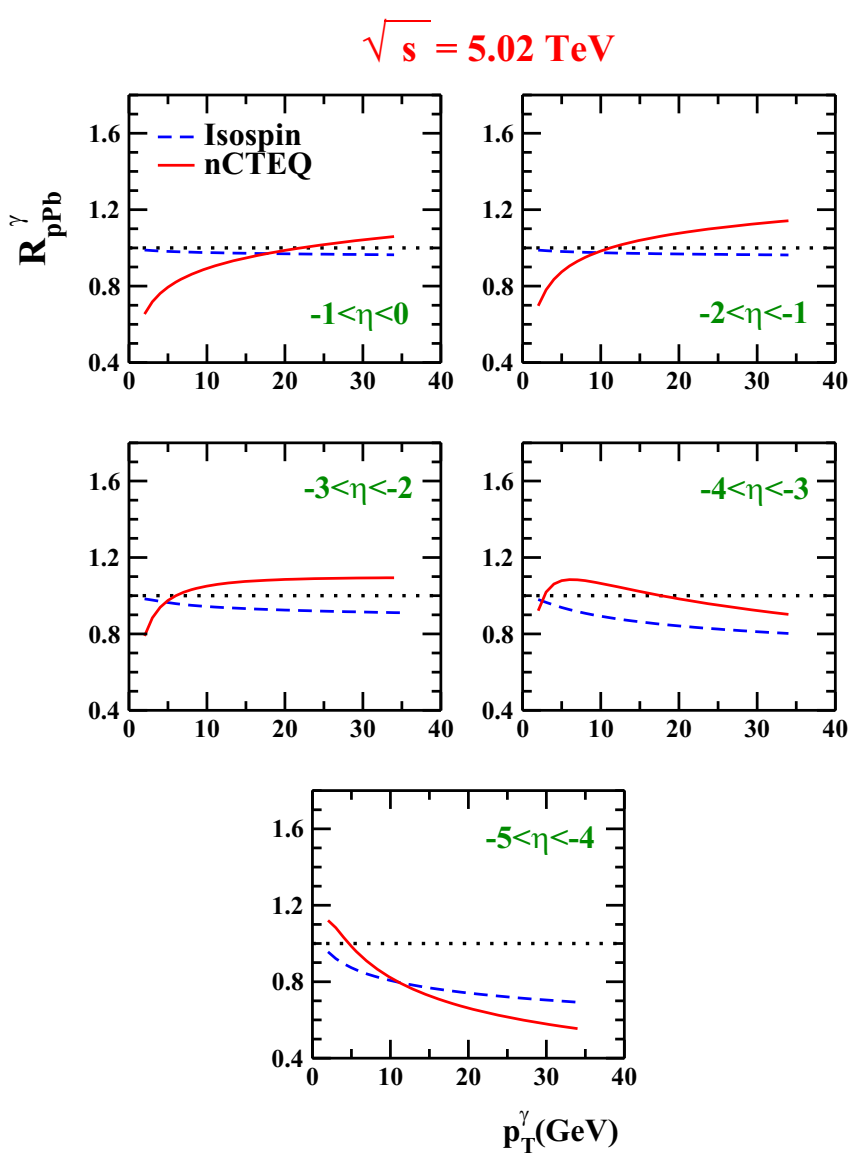

FIG. 3. Same as Fig. 2, but for $\sqrt{s}=5.02 \mathrm{TeV}$.

curve), and the calculation obtained using the nCTEQ15 [28] nuclear modifications (red solid curve) in order to separate the genuine nuclear effects from the sheer isospin effect. According to results obtained for $\sqrt{s}=2.76 \mathrm{TeV}$, as the absolute value of rapidity increases in the backward region, the nuclear modification ratio $R_{p \mathrm{~Pb}}^{\gamma}$ moves from the shadowing area to antishadowing and then the EMC effect. It can be clearly seen that the best kinematic regions for constraining antishadowing nuclear modification are $-2<\eta^{\gamma}<-1$ and $-3<\eta^{\gamma}<-2$, in which the nCTEQ15 prediction lies above the isospin baseline almost in most areas of the $p_{\mathrm{T}}^{\gamma}$ spectrum. To be more precise, at some values of $p_{\mathrm{T}}^{\gamma}$, the difference between the isospin baseline and nCTEQ15 prediction even reaches $20 \%$. It should be noted that the antishadowing nuclear modification can also be investigated from $-1<\eta^{\gamma}<-0$ and $-3<\eta^{\gamma}<$ -4 regions at larger and smaller values of $p_{\mathrm{T}}^{\gamma}$, respectively, but not so well as the case of $-2<\eta^{\gamma}<-1$ and $-3<\eta^{\gamma}<-2$ regions.

Figure 3 shows the same results as Fig. 2, but for the center-of-mass energy of $\sqrt{s}=5.02 \mathrm{TeV}$. As one can see, when the value of $\sqrt{s}$ increases, the cross section and therefore the nuclear modification ratio $R_{p \mathrm{~Pb}}^{\gamma}$ tends towards smaller values of $x$. Therefore, compared to the previous case, moving from the shadowing area towards antishadowing and EMC effect happens somewhat later as the absolute value of rapidity
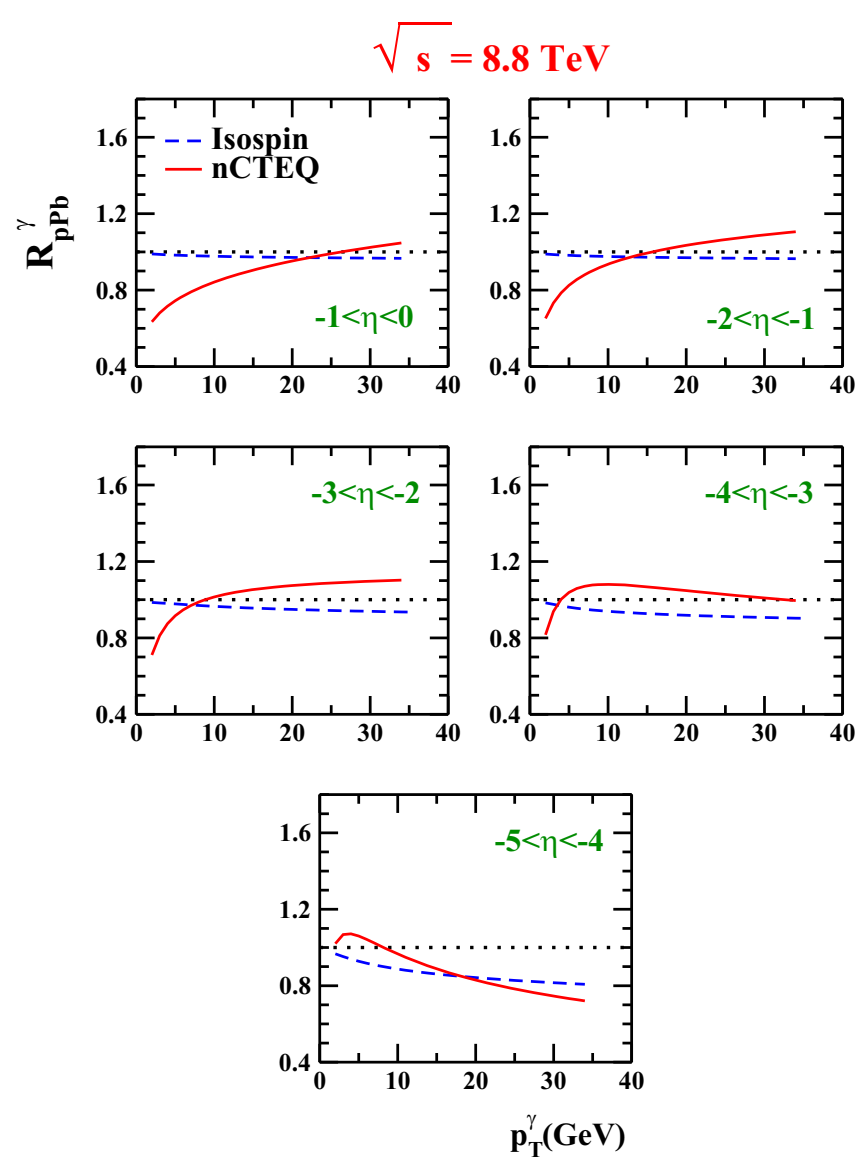

FIG. 4. Same as Fig. 2, but for $\sqrt{s}=8.8 \mathrm{TeV}$.

increases. In this case, the best kinematic regions for constraining antishadowing nuclear modification are $-3<\eta^{\gamma}<$ -2 and $-4<\eta^{\gamma}<-3$.

The corresponding results for $\sqrt{s}=8.8 \mathrm{TeV}$ are shown in Fig. 4. This figure clearly indicates that the best kinematic regions for constraining the antishadowing nuclear modification at such higher energies are again $-3<\eta^{\gamma}<-2$ and $-4<$ $\eta^{\gamma}<-3$, just like the case of $\sqrt{s}=5.02 \mathrm{TeV}$. However, the difference between the isospin baseline and nCTEQ15 prediction in these regions has decreased at $\sqrt{s}=8.8 \mathrm{TeV}$ in comparison with the obtained results at $\sqrt{s}=5.02 \mathrm{TeV}$. Overall, by comparing the three Figs. $2-4$, we can conclude that, among center-of-mass energies considered here, the best one for constraining the antishadowing nuclear modification in backward regions is $\sqrt{s}=2.76 \mathrm{TeV}$. Furthermore, at this energy, among different kinematic regions of photon pseudorapidity, the best regions are $-2<\eta^{\gamma}<-1$ and $-3<\eta^{\gamma}<$ -2 .

\section{B. Rapidity normalized distributions}

As mentioned before, the main advantages of quantity $R_{p \mathrm{~Pb}}^{\gamma}$ introduced in Eq. (5) are its sensitivity to nuclear modifications of parton densities and also some cancellations of the theoretical and experimental uncertainties. However, looking for similar quantities with more benefits is really of interest. It 


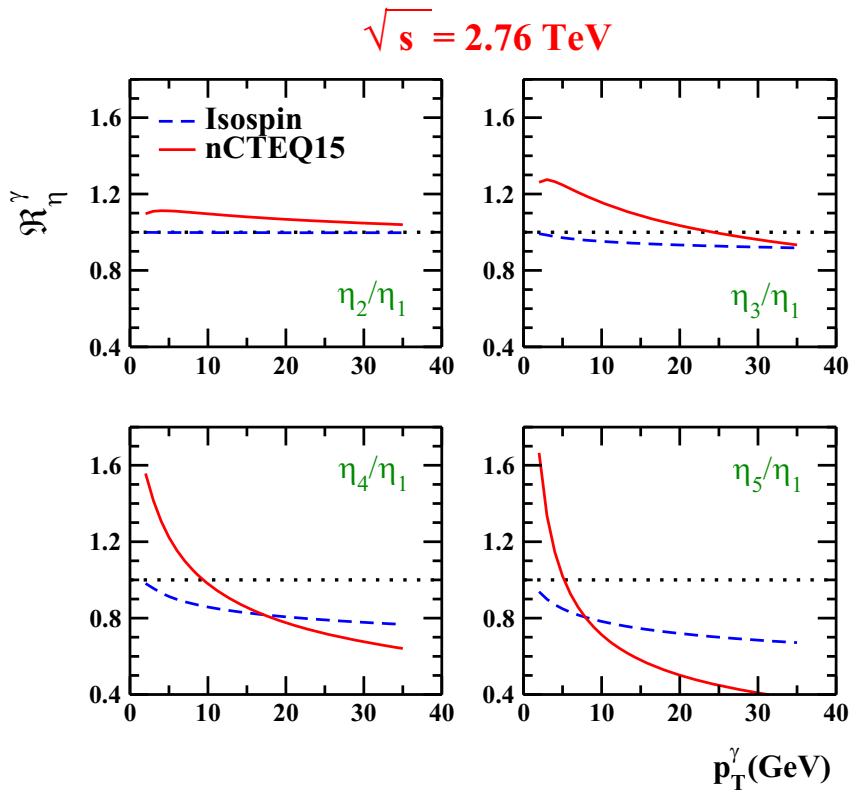

FIG. 5. The rapidity normalized nuclear modification ratio $\mathcal{R}_{\eta}^{\gamma}$ defined in Eq. (6), as a function of $p_{\mathrm{T}}^{\gamma}$ at $\sqrt{s}=2.76 \mathrm{TeV}$ and different rapidity regions, with no nuclear modifications in the PDFs (blue dashed curve) and using the nCTEQ15 [28] nuclear modifications (red solid curve).

has been indicated that the ratio of distributions for different rapidity regions can be a good option [87]. In Refs. [100,101], the authors showed that using rapidity normalized cross section data from heavy flavor production at $\mathrm{LHCb}$ rather than the absolute measurements in a QCD global analysis of PDFs can reduce the scale dependence of the theoretical predictions and then the uncertainties in resulting PDFs. In this section, we are going to examine the possibility of using such quantities to constrain nuclear modifications of PDFs, specially the antishadowing one in our case of study.

According to what was mentioned above, as a first examination we normalize the results obtained for $R_{p \mathrm{~Pb}}^{\gamma}$ in Figs. 2-4 to the corresponding results of the first rapidity bin $-1<$ $\eta^{\gamma}<0$. To be more precise, we define and calculate the rapidity normalized nuclear modification ratio

$$
\mathcal{R}_{\eta, i}^{\gamma} \equiv \frac{\left.R_{p \mathrm{~Pb}}^{\gamma}\right|_{\eta_{i}}}{\left.R_{p \mathrm{~Pb}}^{\gamma}\right|_{\eta_{1}}}
$$

in which $\eta_{i}$, where $i=2,3,4,5$, indicate the rapidity bins $-2<\eta^{\gamma}<-1,-3<\eta^{\gamma}<-2,-4<\eta^{\gamma}<-3$, and $-5<$ $\eta^{\gamma}<-4$, respectively, and $\eta_{1}$ corresponds to bin $-1<\eta^{\gamma}<0$.

The results obtained for different values of center-of-mass energy of $\sqrt{s}=2.76,5.02$, and $8.8 \mathrm{TeV}$ are shown in Figs. 57 , respectively. As can be seen from these figures, there are also some kinematic regions in which the quantity $\mathcal{R}_{\eta}^{\gamma}$ defined in Eq. (6) is sensitive to the antishadowing nuclear modification. In summary, we can say that the best kinematic regions for constraining the antishadowing nuclear modification using the quantity $\mathcal{R}_{\eta}^{\gamma}$ are $\eta_{2} / \eta_{1}$ for $\sqrt{s}=2.76 \mathrm{TeV}$, and also $\eta_{3} / \eta_{1}$ and $\eta_{4} / \eta_{1}$ for $\sqrt{s}=5.02$ and $8.8 \mathrm{TeV}$. For the case of $\sqrt{s}=8.8 \mathrm{TeV}$, the last panel, in which the nuclear

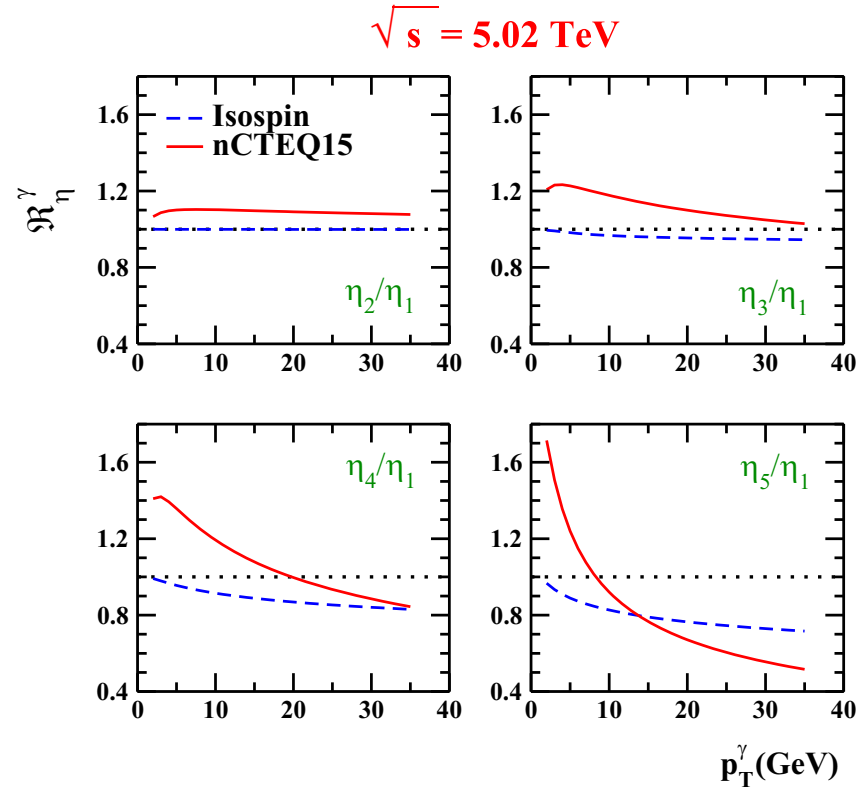

FIG. 6. Same as Fig. 5, but for $\sqrt{s}=5.02 \mathrm{TeV}$.

modification ratio $R_{p \mathrm{~Pb}}^{\gamma}$ for $-5<\eta^{\gamma}<-4$ has been divided bt the one for $-1<\eta^{\gamma}<0$, can be also a good option if we consider the smaller values of $p_{\mathrm{T}}^{\gamma}$. Another point to be noted is that, as the value of center-of-mass energy increases, $\mathcal{R}_{\eta}^{\gamma}$ shows somewhat more sensitivity to the antishadowing nuclear modification. Moreover, note that for the case of $\sqrt{s}=2.76 \mathrm{TeV}$ the last panel shows a good sensitivity to the EMC-effect nuclear modification. Therefore, measuring the quantity $\mathcal{R}_{\eta}^{\gamma}$ in such a kinematic region can also be useful for constraining the EMC-effect nuclear modification.

Although the rapidity normalized nuclear modification ratio $\mathcal{R}_{\eta}^{\gamma}$ can also be used phenomenologically for investigating

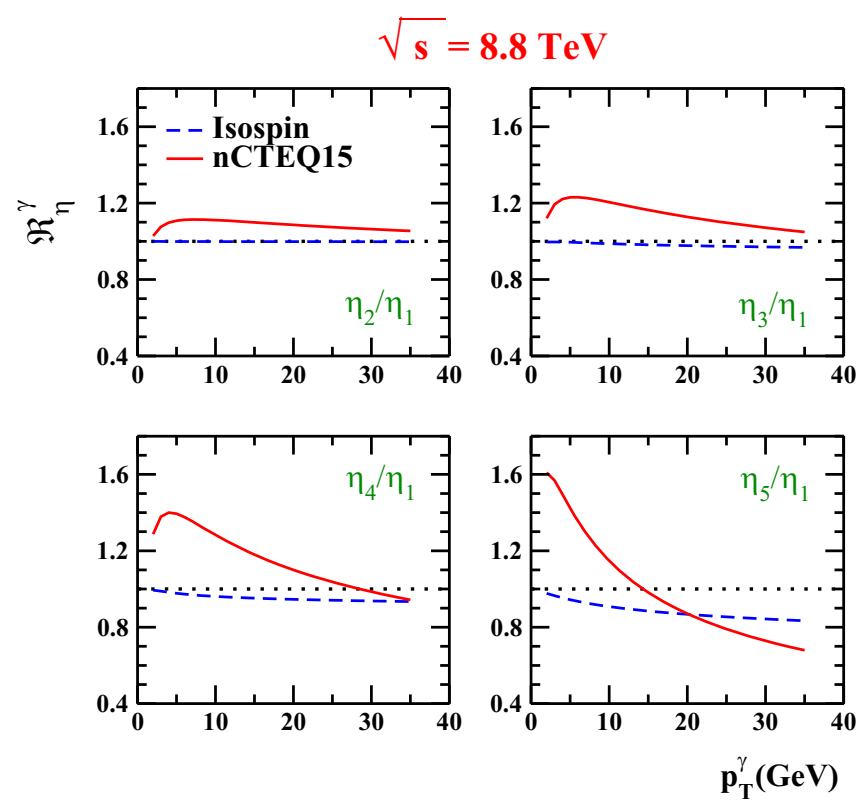

FIG. 7. Same as Fig. 5, but for $\sqrt{s}=8.8 \mathrm{TeV}$. 

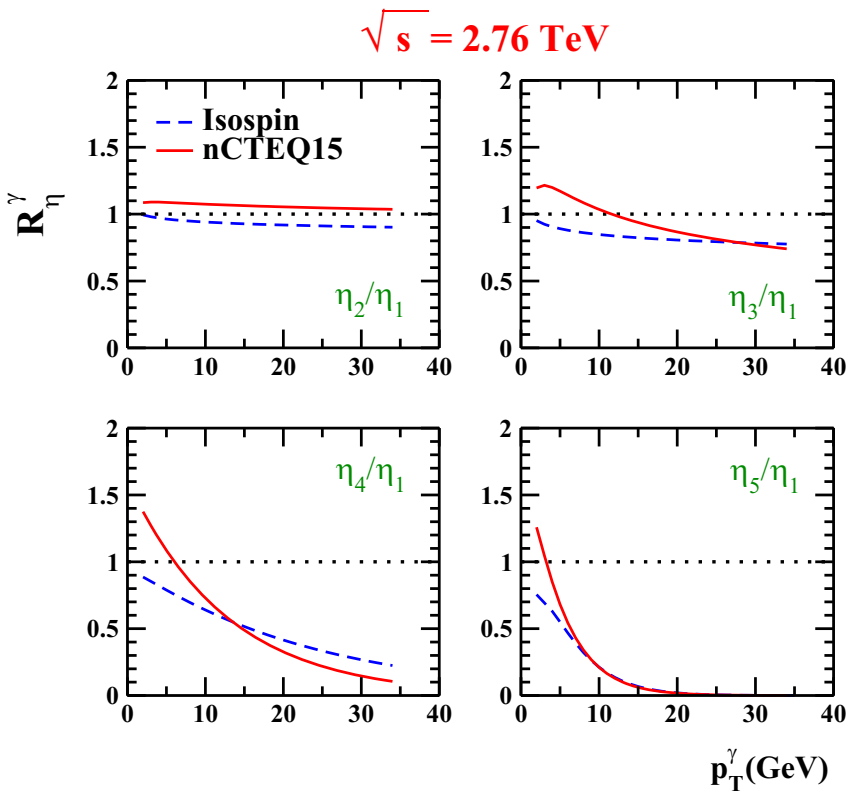

FIG. 8. The ratio of nuclear cross sections for different rapidity regions, $R_{\eta}^{\gamma}$, defined in Eq. (8), as a function of $p_{\mathrm{T}}^{\gamma}$ at $\sqrt{s}=2.76 \mathrm{TeV}$, with no nuclear modifications in the PDFs (blue dashed curve) and using the nCTEQ15 [28] nuclear modifications (red solid curve).

the antishadowing nuclear modification according to results obtained, but it is somewhat complicated experimentally compared to the usual nuclear modification ratio $R_{p \mathrm{~Pb}}^{\gamma}$. Actually, $\mathcal{R}_{\eta}^{\gamma}$ includes two quantities which are expressed in turn as the ratio of cross sections. However, more precisely for this quantity, we find that it can be rewritten as

$$
\mathcal{R}_{\eta, i}^{\gamma} \equiv \frac{\left.R_{p \mathrm{~Pb}}^{\gamma}\right|_{\eta_{i}}}{\left.R_{p \mathrm{~Pb}}^{\gamma}\right|_{\eta_{1}}}=\frac{d \sigma /\left.d p_{\mathrm{T}}\right|_{\eta_{i}} ^{p \mathrm{~Pb}}}{d \sigma /\left.d p_{\mathrm{T}}\right|_{\eta_{1}} ^{p \mathrm{~Pb}}} \times \frac{d \sigma /\left.d p_{\mathrm{T}}\right|_{\eta_{1}} ^{p p}}{d \sigma /\left.d p_{\mathrm{T}}\right|_{\eta_{i}} ^{p p}} .
$$

It is observed that the second fraction on the right side of Eq. (7) does not include any nuclear modification of PDFs and acts as a factor for the first fraction, which includes nuclear modifications in both numerator and denominator. This fact suggests that we can consider the first fraction as a new quantity for exploring the nuclear modification of PDFs. Then, we define the ratio of nuclear cross sections for different rapidity regions as

$$
R_{\eta, i}^{\gamma} \equiv \frac{d \sigma /\left.d p_{\mathrm{T}}\right|_{\eta_{i}} ^{p \mathrm{~Pb}}}{d \sigma /\left.d p_{\mathrm{T}}\right|_{\eta_{1}} ^{p \mathrm{~Pb}}}
$$

where the explanation of indexes is as before. Note that since the nuclear cross sections in the numerator and denominator of Eq. (8) correpsond to different rapidity regions, $R_{\eta}^{\gamma}$ can be totally sensitive to the nuclear modification of PDFs, though some dependencies may be neutralized. The main advantage of $R_{\eta}^{\gamma}$ is that it dose not require a $p p$ baseline measurement with the same $\sqrt{s}$, which is really an important issue.

Actually, from this perspective, $R_{\eta}^{\gamma}$ is similar to the yield asymmetry between the forward and backward rapidities, $Y_{p \mathrm{~Pb}}^{\mathrm{asym}}$, which its usefulness for constraining nuclear PDFs was previously established [38,39,102].
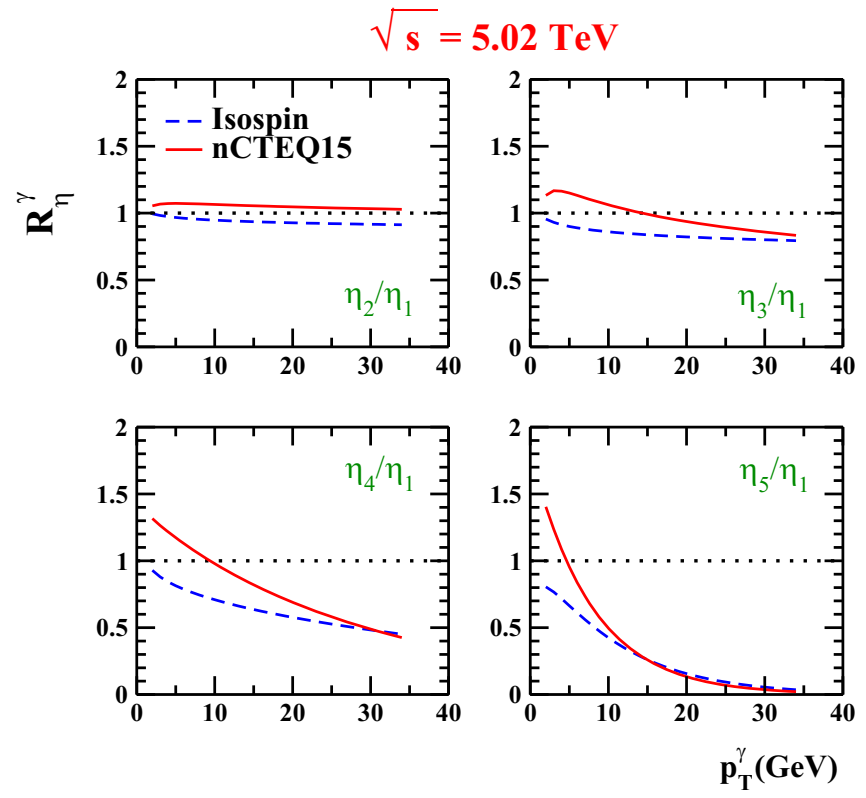

FIG. 9. Same as Fig. 8, but for $\sqrt{s}=5.02 \mathrm{TeV}$.

The results obtained for $R_{\eta}^{\gamma}$ have been shown in Figs. 810 for different values of center-of-mass energy, $\sqrt{s}=2.76$, 5.02 , and $8.8 \mathrm{TeV}$, respectively. Some interesting conclusions can be reached from tehse figures and also by comparing them to the results obtained for $\mathcal{R}_{\eta}^{\gamma}$ in Figs. 5-7. First of all, focusing on each result obtained for $R_{\eta}^{\gamma}$ at various energies, we find that the sensitivity of this quantity to the antishadowing nuclear modification is decreased as the bins with higher value of absolute rapidity are considered in the numerator of $R_{\eta}^{\gamma}$. Moreover, as $p_{\mathrm{T}}^{\gamma}$ increases, $R_{\eta}^{\gamma}$ moves towards the isospin baseline which means that the nuclear modifications in the numerator and denominator of $R_{\eta}^{\gamma}$ have neutralized each other.

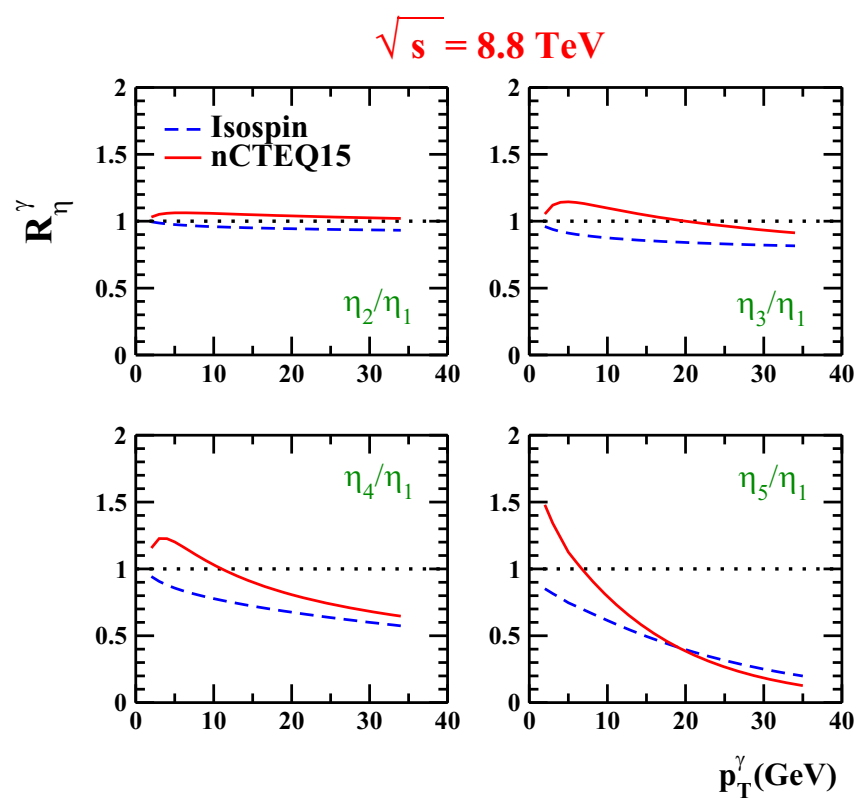

FIG. 10. Same as Fig. 8, but for $\sqrt{s}=8.8 \mathrm{TeV}$. 
By comparing $R_{\eta}^{\gamma}$ and $\mathcal{R}_{\eta}^{\gamma}$ results, one can conclude that they have almost similar behavior, but the curves in $R_{\eta}^{\gamma}$ have shifted downward. This fact indicates that the $p p$ fraction on the right side of Eq. (7) really acts as a factor. Overall, in this case, the best kinematic regions for constraining the antishadowing nuclear modification are $\eta_{2} / \eta_{1}$ for $\sqrt{s}=2.76 \mathrm{TeV}$, and also $\eta_{3} / \eta_{1}$ and $\eta_{4} / \eta_{1}$ for $\sqrt{s}=5.02$ and $8.8 \mathrm{TeV}$. Another interesting point that can be concluded is that the sensitivity of $R_{\eta}^{\gamma}$ to the antishadowing nuclear modification is almost the same as that of $\mathcal{R}_{\eta}^{\gamma}$. Therefore, it can be confirmed that measuring $R_{\eta}^{\gamma}$ for investigating the antishadowing area is experimentally preferable rather than $\mathcal{R}_{\eta}^{\gamma}$ since there is no need to measure the $p p$ cross sections with the same $\sqrt{s}$.

\section{Energy normalized distributions}

Looking for a quantity with more sensitivity to the antishadowing nuclear modification that has the same benefits as before (for example, overall cancellations of the theoretical and experimental uncertainties), in this section we investigate the idea of using the energy normalized distributions. In Ref. [101], the authors investigated the impact of forward charm production data from the LHCb measurements at different center-of-mass energies 5, 7, and $13 \mathrm{TeV}$ on the NNPDF3.0 PDFs [2] and demonstrated that including the cross section ratios between data at different energies in the analysis leads to a reduction in uncertainty of the gluon PDF. Following this idea, as a first step, we normalize the results obtained for $R_{p \mathrm{~Pb}}^{\gamma}$ in Figs. 3 and 4 at $\sqrt{s}=5.02$ and $8.8 \mathrm{TeV}$, respectively, to the corresponding results obtained at $\sqrt{s}=2.76 \mathrm{TeV}$ in Fig. 2 for each rapidity bin, separately. To be more precise, we define and calculate the energy normalized nuclear modification ratio

$$
\mathcal{R}_{s, i}^{\gamma} \equiv \frac{\left.R_{p \mathrm{~Pb}}^{\gamma}\right|_{s_{i}}}{\left.R_{p \mathrm{~Pb}}^{\gamma}\right|_{s_{1}}},
$$

in which $s_{i}$, where $i=1,2$, indicate different center-of-mass energies 5.02 and $8.8 \mathrm{TeV}$, respectively, and $s_{1}$ corresponds to $\sqrt{s}=2.76 \mathrm{TeV}$.

The results obtained for $\mathcal{R}_{s, 1}^{\gamma}$ and $\mathcal{R}_{s, 2}^{\gamma}$ are shown in Figs. 11 and 12 , respectively. As can be seen, in both cases, the quantity $\mathcal{R}_{s}^{\gamma}$ is sensitive to the shadowing nuclear modification in regions with smaller values of absolute rapidity. However, as the rapidity increases in the backward direction, $\mathcal{R}_{s}^{\gamma}$ becomes more sensitive to the antishadowing nuclear modification. To be more precise, in both cases, only last the two panels are useful for constraining the antishadowing nuclear modification. Comparing two Figs. 11 and 12, one can also conclude that, with increasing energy in the numerator of Eq. (9), the sensitivity of $\mathcal{R}_{s}^{\gamma}$ to the shadowing nuclear modification at smaller values of absolute rapidity, and also its sensitivity to the antishadowing nuclear modification at larger values of absolute rapidity, are both intensified. A very interesting point is that for $\mathcal{R}_{s, 2}^{\gamma}$ in rapidity bin $-5<\eta^{\gamma}<-4$, the difference between the nCTEQ15 prediction and isospin baseline even reaches over $25 \%$, which is significant compared to previously considered quantities.

Since the energy normalized nuclear modification ratio $\mathcal{R}_{s}^{\gamma}$, just like the case of the rapidity normalized nuclear
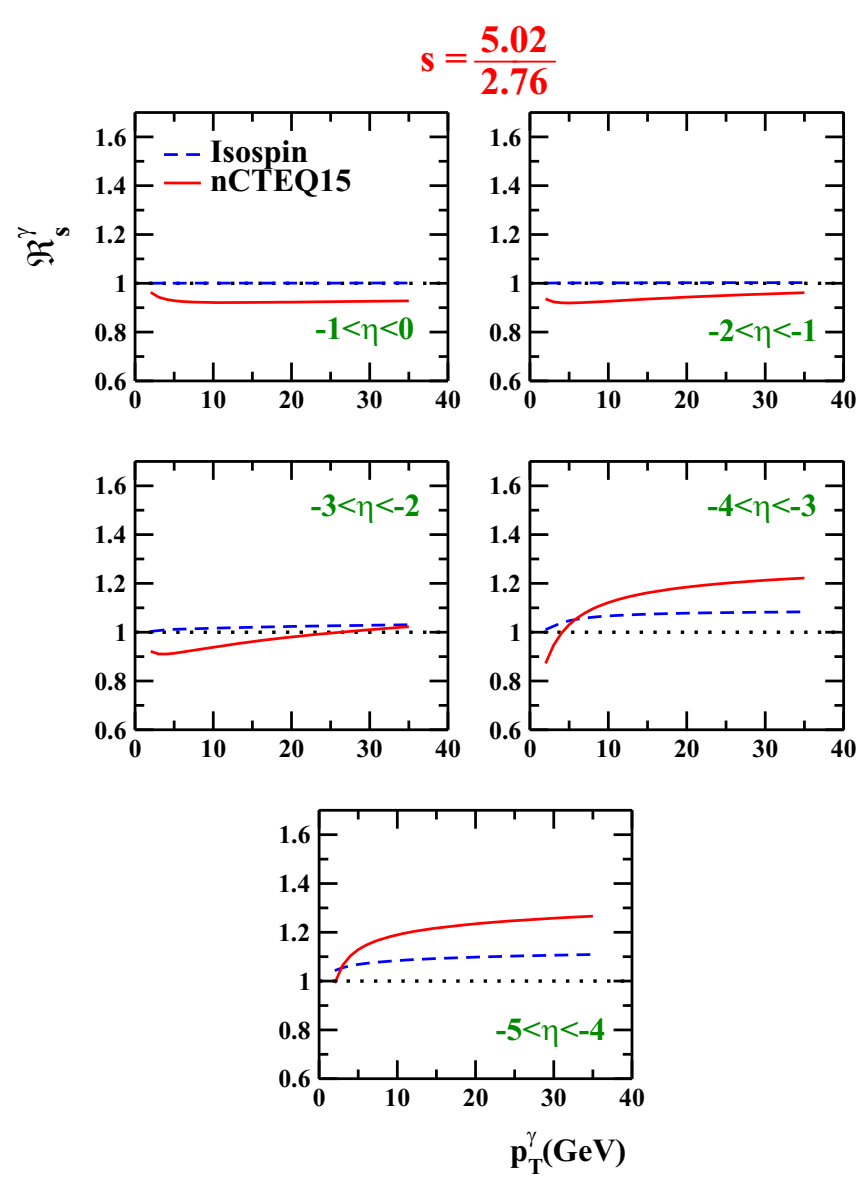

FIG. 11. The energy normalized nuclear modification ratio $\mathcal{R}_{s, 1}^{\gamma}$ defined in Eq. (9), as a function of $p_{\mathrm{T}}^{\gamma}$ for different rapidity regions, with no nuclear modifications in the PDFs (blue dashed curve) and using the nCTEQ15 [28] nuclear modifications (red solid curve).

modification ratio $\mathcal{R}_{\eta}^{\gamma}$ [see Eq. (7)], can be written as a product of two ratios (one containing nuclear cross sections and another without including any nuclear modification), we can remove the $p p$ baseline and define the ratio of nuclear cross sections at different energies as

$$
R_{s, i}^{\gamma} \equiv \frac{d \sigma /\left.d p_{\mathrm{T}}\right|_{s_{i}} ^{\mathrm{pb}}}{d \sigma / d p_{\mathrm{T}} \mid s_{s_{1}}^{\mathrm{Pb}}},
$$

where the explanation of indexes is as before. However, according to the results obtained for $\mathcal{R}_{s}^{\gamma}$, we expect to see some sensitivity to the antishadowing nuclear modification only at higher values of rapidity in the backward direction.

The results obtained for $R_{s, 1}^{\gamma}$ and $R_{s, 2}^{\gamma}$ are shown in Figs. 13 and 14, respectively. Just like the case of $\mathcal{R}_{s}^{\gamma}$, at regions with smaller values of absolute rapidity, the quantities $R_{s, 1}^{\gamma}$ and $R_{s, 2}^{\gamma}$ are sensitive to the shadowing nuclear modification and can be used for constraining the gluon nPDF, which is poorly known in this area. However, as the value of absolute rapidity increases, the spectrum moves towards the isospin baseline and finally some sensitivities to the antishadowing nuclear modification appear in two rapidity bins, $-4<\eta^{\gamma}<-3$ and $-5<\eta^{\gamma}<-4$. Note that unlike $\mathcal{R}_{s}^{\gamma}$, the ratio of nuclear 

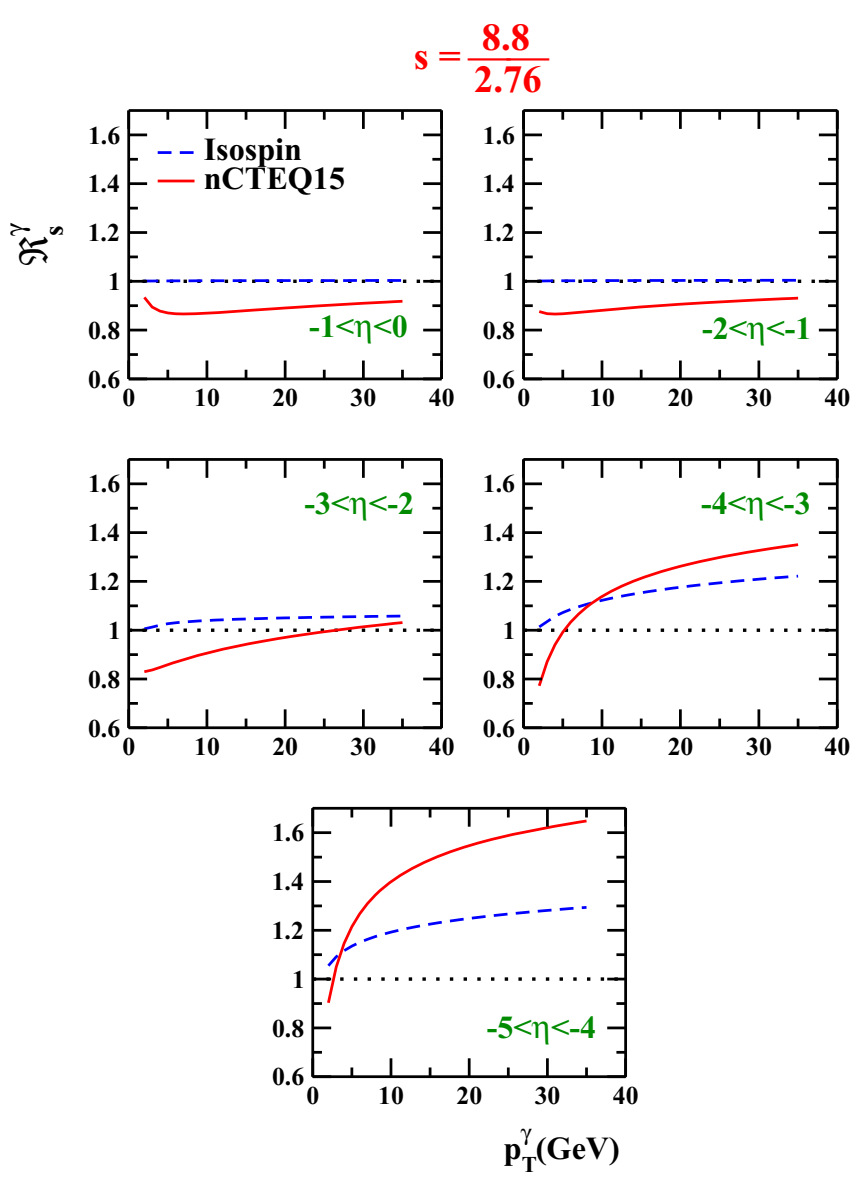

FIG. 12. Same as Fig. 11, but for $\mathcal{R}_{s, 2}^{\gamma}$.

cross sections at different energies $R_{s}^{\gamma}$ follows an exponential behavior as the value of absolute rapidity increases. This fact shows that the ratio of $p p$ cross sections at different energies (in reverse) balances the overall amount of $\mathcal{R}_{s}^{\gamma}$, so that it remains almost around unity (see Figs. 11 and 12). Comparing the results obtained for $R_{s, 1}^{\gamma}$ and $R_{s, 2}^{\gamma}$, we see that they are similar to a large extent in behavior, though their magnitudes are quite different as expected. Overall, we can conclude that the measurement of $R_{s}^{\gamma}$ in the backward direction and at higher values of absolute rapidity and transverse momentum can be very useful for constraining antishadowing nuclear modifications of parton densities, especially of the gluon. Moreover, its measurements is experimentally preferable to $\mathcal{R}_{s}^{\gamma}$, since there is no need to measure the $p p$ cross sections with the same $\sqrt{s}$.

\section{Theoretical uncertainties}

In the previous subsection, we examined various observables to find the best kinematic regions in which the measurement of the isolated prompt photon production in $p-\mathrm{Pb}$ collisions at the LHC has the most sensitivity to the antishadowing area. In all calculations performed so far, we used only nCTEQ15 nPDFs, which have stronger antishadowing nuclear modifications compared with EPS09 [26], DSSZ [27], and HKN07 [24] nPDFs according to Fig. 1. Note also that
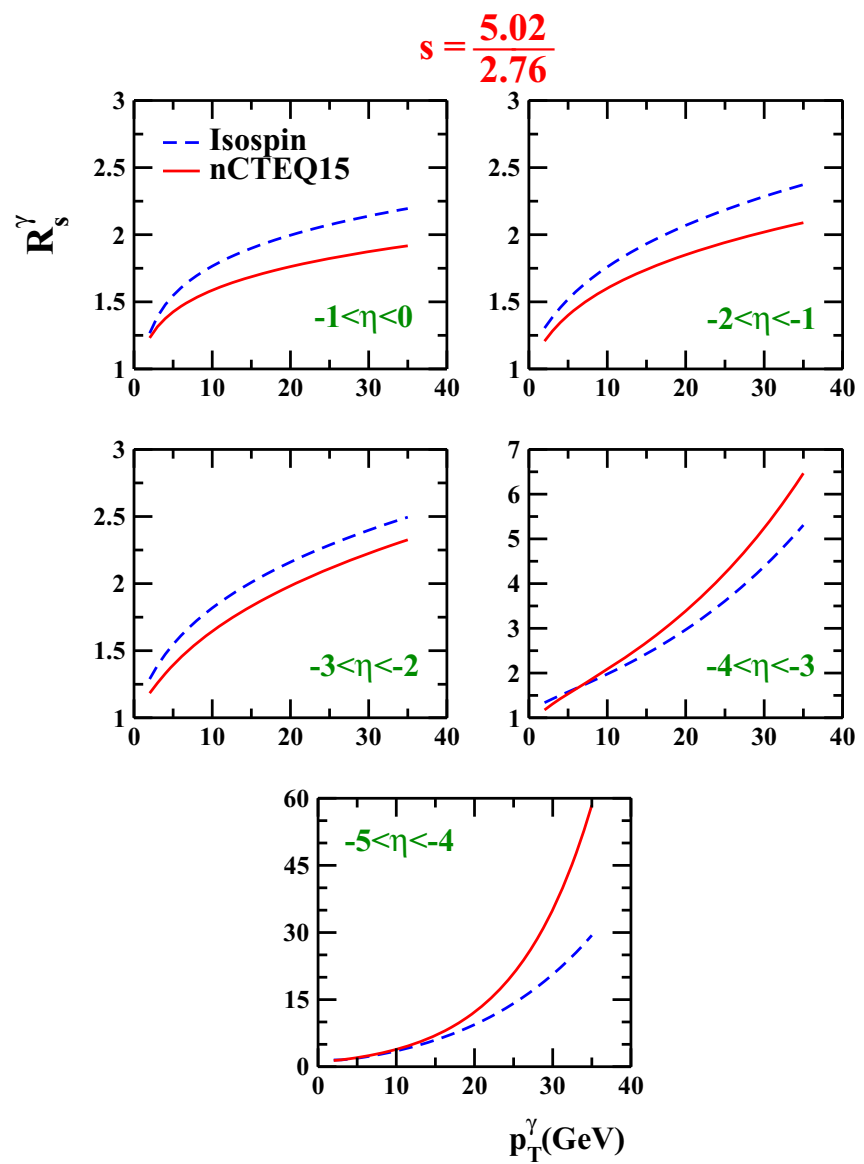

FIG. 13. The ratio of nuclear cross sections at different energies, $R_{s, 1}^{\gamma}$, defined in Eq. (10), as a function of $p_{\mathrm{T}}^{\gamma}$ for different rapidity regions, with no nuclear modifications in the PDFs (blue dashed curve) and using the nCTEQ15 [28] nuclear modifications (red solid curve).

nCTEQ15 has greatest uncertainties in comparison with other groups. As a last step, we now present a comparison between various $\mathrm{nPDFs}$ and also study the theoretical uncertainties in the results due to the nPDFs uncertainties and scale variations. To this aim, we choose the third panel of Fig. 10 in which the ratio of nuclear cross sections for different rapidity regions $\left(\eta_{4} / \eta_{1}\right) R_{\eta}^{\gamma}$ defined in Eq. (8) has been plotted as a function of $p_{\mathrm{T}}^{\gamma}$ at $\sqrt{s}=8.8 \mathrm{TeV}$. As mentioned before, such an observable has the advantage of canceling some experimental and theoretical uncertainties, and also does not require measuring the $p p$ baseline. Note that the theoretical uncertainties of nCTEQ15 nPDFs in any physical quantity such as the cross section can be calculated as usual using the 32 error sets of the nCTEQ15 parametrization (see Ref. [39]).

The results obtained are shown in Fig. 15, where the solid, dotted-dashed, dotted-dashed-dashed, and dotteddotted-dashed curves correspond to the nCTEQ15, EPS09, DSSZ, and HKN07 predictions, respectively, and the red band corresponds to the nCTEQ15 nPDF uncertainties. As before, the isospin baseline has been shown (dashed curve) so that the antishadowing area can be easily distinguished. As can be seen, although the EPS09, DSSZ, and HKN07 predictions 

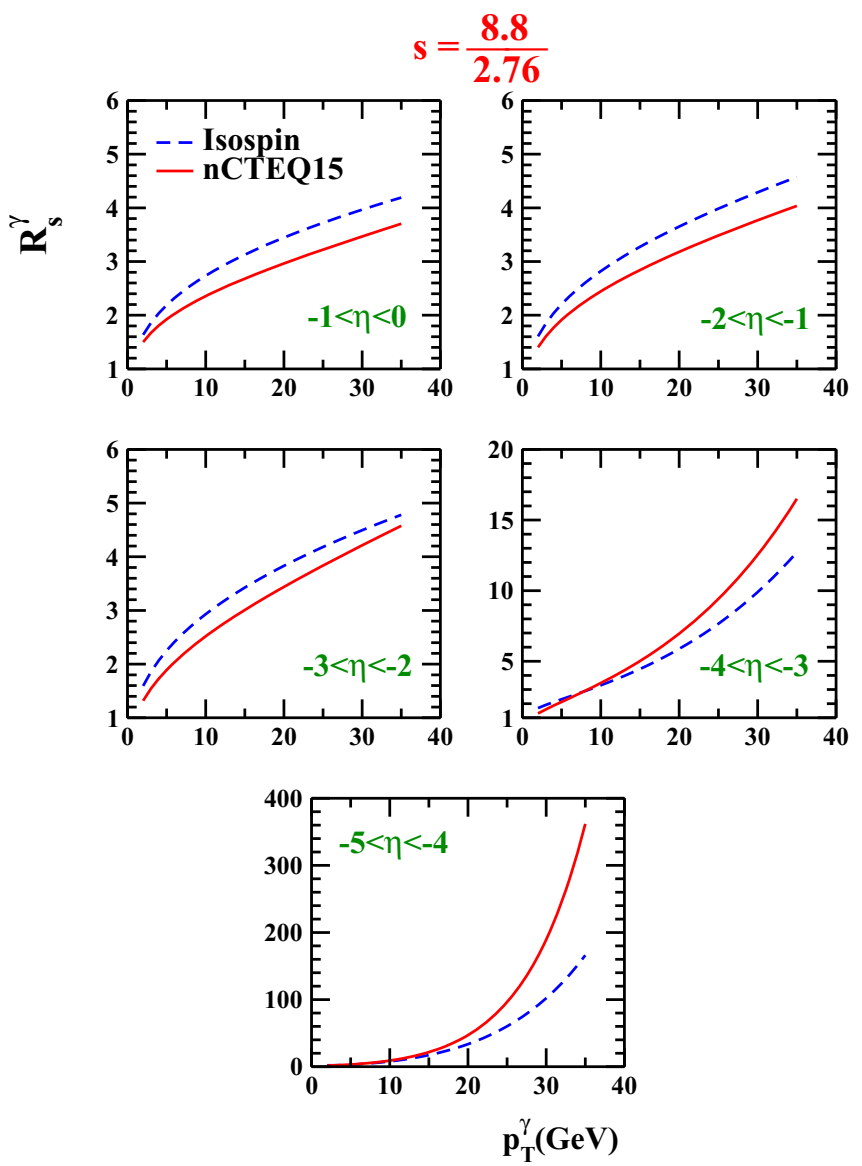

FIG. 14. Same as Fig. 13, but for $R_{s, 2}^{\gamma}$.

are in good agreement with each other, the nCTEQ15 has significant deviations from them, especially at smaller values of $p_{\mathrm{T}}^{\gamma}$. Overall, the EPS09, DSSZ, and HKN07 predictions are placed below the nCTEQ15 prediction in all values of $p_{\mathrm{T}}^{\gamma}$, and are not even within the error band of nCTEQ15 at $p_{\mathrm{T}}^{\gamma}<20 \mathrm{GeV}$. According to the results obtained, one can simply conclude that the measurements of such quantities at the LHC in appropriate kinematics regions can be a very useful tool to accurately determine the antishadowing nuclear modification.

As mentioned before, another important source of the theoretical uncertainties arises from the variation in the renormalization, factorization, and fragmentation scales. In all calculations presented so far, we chose $\mu=M=M_{F}=p_{\mathrm{T}}^{\gamma}$. However, it is also of interest to study the changes in the results due to scale variations. Actually, it can demonstrate the effects of higher order calculations and answer this question: To what extent can the definition of quantities like the ratio of nuclear cross sections for different rapidity regions $R_{n}^{\gamma}$ resolve the lack of higher order calculations? In this regard, we choose again the third panel of Fig. 10 and recompute $R_{\eta}^{\gamma}$ using nCTEQ15 nPDFs, but this time by setting all scales to be equal and varying them by a factor of 2 around the central value $\left(p_{\mathrm{T}}^{\gamma}\right)$.

Figure 16 shows a comparison between the nPDF and scale uncertainties in $R_{\eta}^{\gamma}$ as a function of $p_{\mathrm{T}}^{\gamma}$ at $\sqrt{s}=8.8 \mathrm{TeV}$

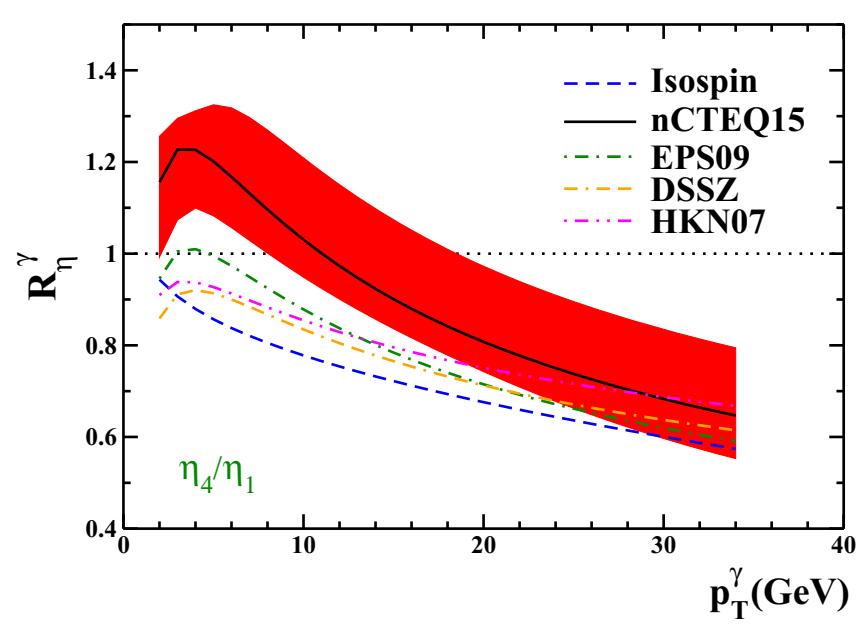

FIG. 15. The ratio of nuclear cross sections for different rapidity regions $\left(\eta_{4} / \eta_{1}\right), R_{\eta}^{\gamma}$, defined in Eq. (8), as a function of $p_{\mathrm{T}}^{\gamma}$ at $\sqrt{s}=8.8 \mathrm{TeV}$, with no nuclear modifications in the PDFs (dashed) and using the nCTEQ15 (solid), EPS09 (dotted-dashed), DSSZ (dotted-dashed-dashed), and HKN07 (dotted-dotted-dashed) nuclear modifications. The red band corresponds to the nCTEQ15 nPDF uncertainties.

and for rapidity region $\eta_{4} / \eta_{1}$. The filled red and hatched green bands represent the nPDF and scale uncertainties, respectively. Note that the black solid curve corresponds to the results obtained using the nCTEQ15 central set. The ratios to the nCTEQ15 central prediction have also been shown in the bottom panel. As can be seen, the nPDF uncertainties are dominant over the scale uncertainties almost in all ranges of

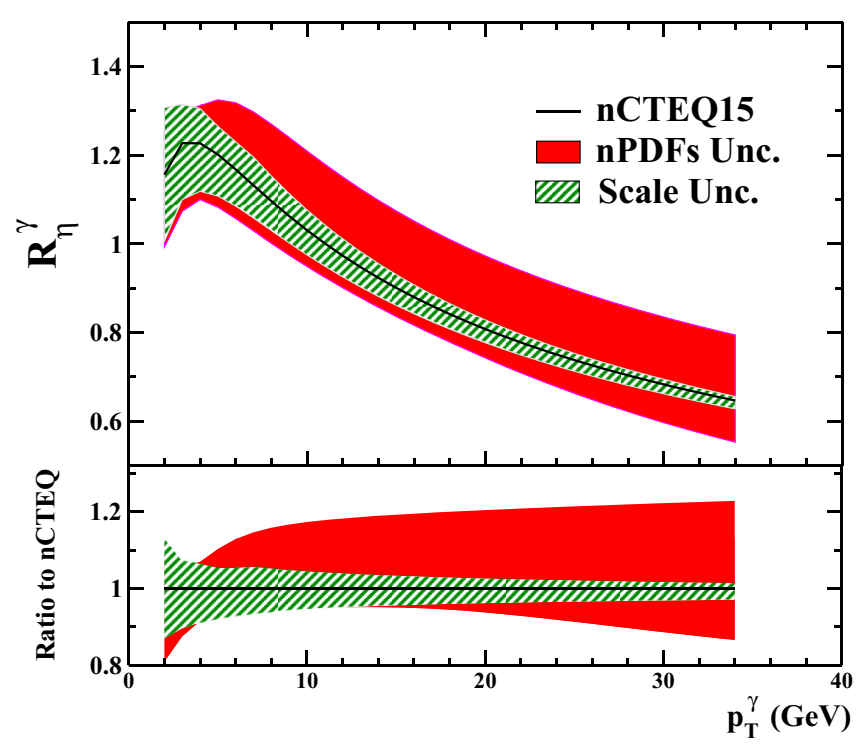

FIG. 16. A comparison between the nPDF and scale uncertainties in $R_{\eta}^{\gamma}$ [see Eq. (8)] as a function of $p_{\mathrm{T}}^{\gamma}$ at $\sqrt{s}=8.8 \mathrm{TeV}$ and for rapidity region $\eta_{4} / \eta_{1}$. The black solid curve corresponds to the results obtained using the nCTEQ15 central set. The filled red and hatched green bands represent the nPDF and scale uncertainties, respectively. The bottom panel shows the ratios to the nCTEQ15 central prediction. 
$p_{\mathrm{T}}^{\gamma}$, except for the smallest values, where the scale uncertainties become very large. This figure clearly demonstrates that the definition of quantities like $R_{\eta}^{\gamma}$ can significantly reduce the scale uncertainties so that they can be of the order of a few percent in some kinematics regions and therefore negligible compared with the nPDF uncertainties.

\section{SUMMARY AND CONCLUSIONS}

The prompt photon production in hadronic collisions has been an interesting subject of research for more than three decades, since it provides a powerful tool for testing perturbative QCD and brings useful information on the gluon PDF and also its nuclear modification. Because of the lack of experimental data on nuclei in the initial state compared with the case of free nucleons which are suitable for use in a global analysis, our knowledge of nuclear modifications of parton densities is not as good as for PDFs. In this work, we performed a comprehensive study of the isolated prompt photon production in $p$ - $\mathrm{Pb}$ collisions at backward rapidities to find how its measurements can put constraints on the antishadowing nuclear modification of the gluon PDF. In this regard, we calculated and compared various quantities, including the nuclear modification ratio, the rapidity and energy normalized nuclear modification ratios, and the ratio of nuclear cross sections for different rapidity regions, and also different values of center-of-mass energy covered by the LHC to realize which one is most useful. For the case of the nuclear modification ratio $R_{p \mathrm{~Pb}}^{\gamma}$, we found that as the absolute value of rapidity increases in the backward direction, it moves from the shadowing area to antishadowing and then to the EMC effect. We showed that among center-of-mass energies $\sqrt{s}=$ 2.76, 5.02, 8.8 TeV, the best one for constraining the antishadowing nuclear modification using $R_{p \mathrm{~Pb}}^{\gamma}$ is the lowest one. Furthermore, at this energy, among different kinematic regions of photon pseudorapidity, the best regions are $-2<\eta^{\gamma}<-1$ and $-3<\eta^{\gamma}<-2$. For the case of rapidity normalized nuclear modification ratio $\mathcal{R}_{\eta}^{\gamma}$, we demonstrated that as the value of center-of-mass energy increases, it shows somewhat more sensitivity to tje antishadowing nuclear modification, so that the best kinematic regions for constraining antishadowing are $\eta_{3} / \eta_{1}$ and $\eta_{4} / \eta_{1}$ for $\sqrt{s}=8.8 \mathrm{TeV}$. Meanwhile, we found that measuring $\mathcal{R}_{\eta}^{\gamma}$ at lower center-of-mass energy $\sqrt{s}=2.76 \mathrm{TeV}$ and rapidity region $\eta_{5} / \eta_{1}$ can be also used for constraining the EMC-effect nuclear modification. Then, we calculated the ratio of nuclear cross sections for different rapidity regions $R_{\eta}^{\gamma}$ at various energies. We found that the sensitivity of this quantity to the antishadowing nuclear modification decreases as the bins with higher value of absolute rapidity are considered. We indicated that $R_{\eta}^{\gamma}$ and $\mathcal{R}_{\eta}^{\gamma}$ have almost similar behavior, but the curves in $R_{\eta}^{\gamma}$ are shifted downward. However, the sensitivity of $R_{\eta}^{\gamma}$ to the antishadowing nuclear modification is almost the same as that of $\mathcal{R}_{\eta}^{\gamma}$. Therefore, we concluded that measuring $R_{\eta}^{\gamma}$ is experimentally preferable to $\mathcal{R}_{\eta}^{\gamma}$ since there is no need to measure the $p p$ cross sections with the same $\sqrt{s}$. For the case of the energy normalized nuclear modification ratio $\mathcal{R}_{s}^{\gamma}$, we show that it is sensitive to the shadowing nuclear modification at regions with smaller values of absolute rapidity. However, as the rapidity increases in the backward direction, $\mathcal{R}_{s}^{\gamma}$ becomes more sensitive to the antishadowing nuclear modification, so that the best kinematic regions are $-4<\eta^{\gamma}<-3$ and $-5<\eta^{\gamma}<-4$. Moreover, we found that with increasing energy in the numerator of $\mathcal{R}_{s}^{\gamma}$, its sensitivity to the shadowing and antishadowing nuclear modifications at smaller and larger values of absolute rapidity, respectively, is intensified in both cases. Finally, we calculated the ratio of nuclear cross sections at different energies, $R_{s}^{\gamma}$, and found that it behaves to a large extent as $\mathcal{R}_{s}^{\gamma}$, though its magnitude is quite different. To be more precise, it can be used for constraining the shadowing and antishadowing nuclear modifications at smaller and larger values of absolute rapidity, respectively. However, we confirm that its measurement in the backward direction and at higher values of absolute rapidity and transverse momentum is experimentally preferable to $\mathcal{R}_{s}^{\gamma}$ for constraining the antishadowing area, since there is no need to measure the $p p$ cross sections with the same $\sqrt{s}$. We also presented a comparison between various nPDFs and also studied the theoretical uncertainties due to the nPDF uncertainties and scale variations. We showed that, for $R_{\eta}^{\gamma}$ as an example, the EPS09, DSSZ, and HKN07 predictions are in good agreement with each other, but the nCTEQ15 has significant deviations from them, especially for smaller values of $p_{\mathrm{T}}^{\gamma}$. We concluded that the measurements of such quantities at the LHC in appropriate kinematics regions can be a very useful tool to accurately determine the antishadowing nuclear modification. Moreover, we demonstrated that the nPDF uncertainties are dominant over scale uncertainties almost in all ranges of $p_{\mathrm{T}}^{\gamma}$. We confirm that the definition of quantities like $R_{\eta}^{\gamma}$ can significantly reduce the scale uncertainties, so that they can be of the order of a few percent in some kinematics regions and therefore negligible compared with the nPDF uncertainties.

\section{ACKNOWLEDGMENTS}

The authors thank Hamzeh Khanpour for useful discussions and comments. M.G. also thanks the School of Particles and Accelerators, Institute for Research in Fundamental Sciences (IPM) for financial support provided for this research. S.R. is grateful to CERN-TH division for their warm hospitality.
[1] A. Accardi, L. T. Brady, W. Melnitchouk, J. F. Owens, and N. Sato, Constraints on large- $x$ parton distributions from new weak boson production and deep-inelastic scattering data, Phys. Rev. D 93, 114017 (2016).

[2] R. D. Ball et al. (NNPDF Collaboration), Parton distributions for the LHC Run II, J. High Energy Phys. 04 (2015) 040.
[3] L. A. Harland-Lang, A. D. Martin, P. Motylinski, and R. S. Thorne, Parton distributions in the LHC era: MMHT 2014 PDFs, Eur. Phys. J. C 75, 204 (2015).

[4] P. Jimenez-Delgado and E. Reya, Delineating parton distributions and the strong coupling, Phys. Rev. D 89, 074049 (2014). 
[5] S. Dulat et al., New parton distribution functions from a global analysis of quantum chromodynamics, Phys. Rev. D 93, 033006 (2016).

[6] A. Aleedaneshvar, M. Goharipour, and S. Rostami, Uncertainty of parton distribution functions due to physical observables in a global analysis, Chin. Phys. C 41, 023101 (2017).

[7] S. Alekhin, J. Blümlein, S. Moch, and R. Plačakytè, Parton distribution functions, $\alpha_{s}$, and heavy-quark masses for LHC Run II, Phys. Rev. D 96, 014011 (2017).

[8] R. D. Ball et al. (NNPDF Collaboration), Parton distributions from high-precision collider data, Eur. Phys. J. C 77, 663 (2017).

[9] S. M. M. Nejad, H. Khanpour, S. A. Tehrani, and M. Mahdavi, QCD analysis of nucleon structure functions in deep-inelastic neutrino-nucleon scattering: Laplace transform and Jacobi polynomials approach, Phys. Rev. C 94, 045201 (2016).

[10] H. Khanpour, A. Mirjalili, and S. A. Tehrani, Analytic derivation of the next-to-leading order proton structure function $F_{2}^{p}\left(x, Q^{2}\right)$ based on the Laplace transformation, Phys. Rev. C 95, 035201 (2017).

[11] J. Butterworth et al., PDF4LHC recommendations for LHC Run II, J. Phys. G 43, 023001 (2016).

[12] P. Jimenez-Delgado et al. (Jefferson Lab Angular Momentum (JAM) Collaboration), Constraints on spin-dependent parton distributions at large $x$ from global QCD analysis, Phys. Lett. B 738, 263 (2014).

[13] N. Sato, W. Melnitchouk, S. E. Kuhn, J. J. Ethier, and A. Accardi (Jefferson Lab Angular Momentum Collaboration), Iterative Monte Carlo analysis of spin-dependent parton distributions, Phys. Rev. D 93, 074005 (2016).

[14] F. Taghavi-Shahri, H. Khanpour, S. Atashbar Tehrani, and Z. Alizadeh Yazdi, Next-to-next-to-leading order QCD analysis of spin-dependent parton distribution functions and their uncertainties: Jacobi polynomials approach, Phys. Rev. D 93, 114024 (2016).

[15] H. Khanpour, S. Taheri Monfared, and S. Atashbar Tehrani, Nucleon spin structure functions at NNLO in the presence of target mass corrections and higher twist effects, Phys. Rev. D 95, 074006 (2017).

[16] J. J. Ethier, N. Sato, and W. Melnitchouk, First Simultaneous Extraction of Spin-Dependent Parton Distributions and Fragmentation Functions from a Global QCD Analysis, Phys. Rev. Lett. 119, 132001 (2017).

[17] H. Khanpour, S. T. Monfared, and S. A. Tehrani, Study of spin-dependent structure functions of ${ }^{3} \mathrm{He}$ and ${ }^{3} \mathrm{H}$ at NNLO approximation and corresponding nuclear corrections, Phys. Rev. D 96, 074037 (2017).

[18] M. Salajegheh, S. M. Nejad, H. Khanpour, and S. A. Tehrani, Analytical approaches to the determination of spin-dependent parton distribution functions at NNLO approximation, Phys. Rev. C 97, 055201 (2018).

[19] S. M. Moosavi Nejad, M. Soleymaninia, and A. Maktoubian, Proton fragmentation functions considering finite-mass corrections, Eur. Phys. J. A 52, 316 (2016).

[20] E. Leader, A. V. Sidorov, and D. B. Stamenov, Determination of the fragmentation functions from an NLO QCD analysis of the HERMES data on pion multiplicities, Phys. Rev. D 93, 074026 (2016).

[21] D. de Florian, M. Epele, R. J. Hernandez-Pinto, R. Sassot, and M. Stratmann, Parton-to-kaon fragmentation revisited, Phys. Rev. D 95, 094019 (2017).
[22] M. Soleymaninia, H. Khanpour, and S. M. Nejad, First determination of $D^{*+}$-meson fragmentation functions and their uncertainties at next-to-next-to-leading order, Phys. Rev. D 97, 074014 (2018).

[23] M. Soleymaninia, M. Goharipour, and H. Khanpour, First global QCD analysis of charged hadron fragmentation functions and their uncertainties at next-to-next-to-leading order, Phys. Rev. D 98, 074002 (2018).

[24] M. Hirai, S. Kumano, and T.-H. Nagai, Determination of nuclear parton distribution functions and their uncertainties in next-to-leading order, Phys. Rev. C 76, 065207 (2007).

[25] I. Schienbein, J. Y. Yu, K. Kovarik, C. Keppel, J. G. Morfin, F. I. Olness, and J. F. Owens, PDF nuclear corrections for charged and neutral current processes, Phys. Rev. D 80, 094004 (2009).

[26] K. J. Eskola, H. Paukkunen, and C. A. Salgado, EPS09: A new generation of NLO and LO nuclear parton distribution functions, J. High Energy Phys. 04 (2009) 065.

[27] D. de Florian, R. Sassot, P. Zurita, and M. Stratmann, Global analysis of nuclear parton distributions, Phys. Rev. D 85, 074028 (2012).

[28] K. Kovarik et al., nCTEQ15: Global analysis of nuclear parton distributions with uncertainties in the CTEQ framework, Phys. Rev. D 93, 085037 (2016).

[29] H. Khanpour and S. Atashbar Tehrani, Global analysis of nuclear parton distribution functions and their uncertainties at next-to-next-to-leading order, Phys. Rev. D 93, 014026 (2016).

[30] K. J. Eskola, P. Paakkinen, H. Paukkunen, and C. A. Salgado, EPPS16: Nuclear parton distributions with LHC data, Eur. Phys. J. C 77, 163 (2017).

[31] R. Wang, X. Chen, and Q. Fu, Global study of nuclear modifications on parton distribution functions, Nucl. Phys. B 920, 1 (2017).

[32] J. C. Collins, D. E. Soper, and G. F. Sterman, Factorization of hard processes in QCD, Adv. Ser. Direct. High Energy Phys. 5, 1 (1989).

[33] R. Brock et al. (CTEQ Collaboration), Handbook of perturbative QCD: Version 1.0, Rev. Mod. Phys. 67, 157 (1995).

[34] F. Arleo and T. Gousset, Measuring gluon shadowing with prompt photons at RHIC and LHC, Phys. Lett. B 660, 181 (2008).

[35] C. Brenner Mariotto and V. P. Goncalves, Nuclear shadowing and prompt photons in hadronic collisions at ultrarelativistic energies, Phys. Rev. C 78, 037901 (2008).

[36] L. J. Zhou, H. Zhang, and E. Wang, Shadowing effects in direct photon and hadron production in heavy-ion collisions, J. Phys. G 37, 105109 (2010).

[37] F. Arleo, K. J. Eskola, H. Paukkunen, and C. A. Salgado, Inclusive prompt photon production in nuclear collisions at RHIC and LHC, J. High Energy Phys. 04 (2011) 055.

[38] I. Helenius, K. J. Eskola, and H. Paukkunen, Probing the small- $x$ nuclear gluon distributions with isolated photons at forward rapidities in $\mathrm{p}+\mathrm{Pb}$ collisions at the LHC, J. High Energy Phys. 09 (2014) 138.

[39] M. Goharipour and H. Mehraban, Study of isolated prompt photon production in $p$ - $\mathrm{Pb}$ collisions for the ALICE kinematics, Phys. Rev. D 95, 054002 (2017).

[40] M. Klasen, K. Kovarik, and J. Potthoff, Nuclear parton density functions from jet production in DIS at the EIC, Phys. Rev. D 95, 094013 (2017). 
[41] M. Klasen and K. Kovařík, Nuclear parton density functions from dijet photoproduction at the EIC, Phys. Rev. D 97, 114013 (2018).

[42] M. Bury, H. Van Haevermaet, A. Van Hameren, P. Van Mechelen, K. Kutak, and M. Serino, Single inclusive jet production and the nuclear modification ratio at very forward rapidity in proton-lead collisions with $\sqrt{s_{N N}}=5.02 \mathrm{TeV}$, Phys. Lett. B 780, 185 (2018).

[43] A. Kusina, J. P. Lansberg, I. Schienbein, and H. S. Shao, Gluon Shadowing in Heavy-Flavor Production at the LHC, Phys. Rev. Lett. 121, 052004 (2018).

[44] P. Aurenche, A. Douiri, R. Baier, M. Fontannaz, and D. Schiff, Prompt photon production at large $p_{\mathrm{T}}$ in QCD beyond the leading order, Phys. Lett. B 140, 87 (1984).

[45] J. F. Owens, Large momentum transfer production of direct photons, jets, and particles, Rev. Mod. Phys. 59, 465 (1987).

[46] J. Huston, E. Kovacs, S. Kuhlmann, H. L. Lai, J. F. Owens, and W. K. Tung, Global QCD study of direct photon production, Phys. Rev. D 51, 6139 (1995).

[47] P. Aurenche, M. Fontannaz, J. P. Guillet, B. A. Kniehl, E. Pilon, and M. Werlen, A critical phenomenological study of inclusive photon production in hadronic collisions, Eur. Phys. J. C 9, 107 (1999).

[48] P. Aurenche, J. P. Guillet, E. Pilon, M. Werlen, and M. Fontannaz, New critical study of photon production in hadronic collisions, Phys. Rev. D 73, 094007 (2006).

[49] J. Alam, S. Sarkar, P. Roy, T. Hatsuda, and B. Sinha, Thermal photons and lepton pairs from quark gluon plasma and hot hadronic matter, Ann. Phys. (NY) 286, 159 (2001).

[50] E. V. Shuryak, Quantum chromodynamics and the theory of superdense matter, Phys. Rep. 61, 71 (1980).

[51] D. G. d'Enterria, Quark-gluon matter, J. Phys. G 34, S53 (2007).

[52] J. F. Paquet, C. Shen, G. Denicol, M. Luzum, B. Schenke, S. Jeon, and C. Gale, Thermal and prompt photons at RHIC and the LHC, Nucl. Phys. A 956, 409 (2016).

[53] P. Aurenche, R. Baier, M. Fontannaz, J. F. Owens, and M. Werlen, Gluon contents of the nucleon probed with real and virtual photons, Phys. Rev. D 39, 3275 (1989).

[54] W. Vogelsang and A. Vogt, Constraints on the proton's gluon distribution from prompt photon production, Nucl. Phys. B 453, 334 (1995).

[55] R. Ichou and D. d'Enterria, Sensitivity of isolated photon production at $\mathrm{TeV}$ hadron colliders to the gluon distribution in the proton, Phys. Rev. D 82, 014015 (2010).

[56] D. d'Enterria and J. Rojo, Quantitative constraints on the gluon distribution function in the proton from collider isolatedphoton data, Nucl. Phys. B 860, 311 (2012).

[57] J. M. Campbell, J. Rojo, E. Slade, and C. Williams, Direct photon production and PDF fits reloaded, Eur. Phys. J. C 78 , 470 (2018).

[58] V. A. Bednyakov, M. A. Demichev, G. I. Lykasov, T. Stavreva, and M. Stockton, Searching for intrinsic charm in the proton at the LHC, Phys. Lett. B 728, 602 (2014).

[59] S. Rostami, M. Goharipour, and A. Aleedaneshvar, Role of the intrinsic charm content of the nucleon from various lightcone models on $\gamma+\mathrm{c}$-jet production, Chin. Phys. C 40, 123104 (2016).

[60] S. S. Adler et al. (PHENIX Collaboration), Centrality Dependence of Direct Photon Production in $\sqrt{s_{N N}}=200 \mathrm{GeV} \mathrm{Au}+$ Au Collisions, Phys. Rev. Lett. 94, 232301 (2005).
[61] S. Afanasiev et al. (PHENIX Collaboration), Measurement of Direct Photons in $\mathrm{Au}+\mathrm{Au}$ Collisions at $\sqrt{s_{N N}}=200 \mathrm{GeV}$, Phys. Rev. Lett. 109, 152302 (2012).

[62] A. Adare et al. (PHENIX Collaboration), Enhanced Production of Direct Photons in Au+Au Collisions at $\sqrt{s_{N N}}=200$ $\mathrm{GeV}$ and Implications for the Initial Temperature, Phys. Rev. Lett. 104, 132301 (2010).

[63] A. Adare et al. (PHENIX Collaboration), Centrality dependence of low-momentum direct-photon production in $\mathrm{Au}+$ $\mathrm{Au}$ collisions at $\sqrt{s_{N N}}=200 \mathrm{GeV}$, Phys. Rev. C 91, 064904 (2015).

[64] A. Adare et al. (PHENIX Collaboration), Direct photon production in $d+\mathrm{Au}$ collisions at $\sqrt{s_{N N}}=200 \mathrm{GeV}$, Phys. Rev. C 87, 054907 (2013).

[65] M. Wilde (ALICE Collaboration), Measurement of direct photons in $\mathrm{pp}$ and $\mathrm{Pb}-\mathrm{Pb}$ Collisions with ALICE, Nucl. Phys. A 904-905, 573c (2013).

[66] J. Adam et al. (ALICE Collaboration), Direct photon production in $\mathrm{Pb}-\mathrm{Pb}$ collisions at $\sqrt{s_{N N}}=2.76 \mathrm{TeV}$, Phys. Lett. B 754, 235 (2016).

[67] G. Aad et al. (ATLAS Collaboration), Centrality, rapidity and transverse momentum dependence of isolated prompt photon production in lead-lead collisions at $\sqrt{s_{N N}}=2.76 \mathrm{TeV}$ measured with the ATLAS detector, Phys. Rev. C 93, 034914 (2016).

[68] S. Chatrchyan et al. (CMS Collaboration), Measurement of isolated photon production in $p p$ and $\mathrm{PbPb}$ collisions at $\sqrt{s_{N N}}=2.76 \mathrm{TeV}$, Phys. Lett. B 710, 256 (2012).

[69] M. Aaboud et al. (ATLAS collaboration), Measurement of prompt photon production in $\sqrt{s_{\mathrm{NN}}}=8.16 \mathrm{TeV} p+\mathrm{Pb}$ collisions with ATLAS, arXiv:1903.02209.

[70] P. Paakkinen, Nuclear parton distribution functions, Frascati Phys. Ser., 33 (2017), arXiv:1802.05927.

[71] P. Aurenche, R. Baier, M. Fontannaz, and D. Schiff, Prompt photon production at large $p_{\mathrm{T}}$ scheme invariant QCD predictions and comparison with experiment, Nucl. Phys. B 297, 661 (1988).

[72] P. Aurenche, R. Baier, and M. Fontannaz, Prompt photon production at colliders, Phys. Rev. D 42, 1440 (1990).

[73] H. Baer, J. Ohnemus and J. F. Owens, Next-to-leading logarithm calculation of direct photon production, Phys. Rev. D 42, 61 (1990).

[74] E. L. Berger and J. Qiu, Calculations of prompt photon production in QCD, Phys. Rev. D 44, 2002 (1991).

[75] L. E. Gordon and W. Vogelsang, Polarized and unpolarized isolated prompt photon production beyond the leading order, Phys. Rev. D 50, 1901 (1994).

[76] M. Fontannaz, J. P. Guillet, and G. Heinrich, Isolated prompt photon photoproduction at NLO, Eur. Phys. J. C 21, 303 (2001).

[77] S. Catani, M. Fontannaz, J. P. Guillet, and E. Pilon, Crosssection of isolated prompt photons in hadron-hadron collisions, J. High Energy Phys. 05 (2002) 028.

[78] Z. Belghobsi, M. Fontannaz, J.-P. Guillet, G. Heinrich, E. Pilon, and M. Werlen, Photon-jet correlations and constraints on fragmentation functions, Phys. Rev. D 79, 114024 (2009).

[79] I. Helenius, K. J. Eskola, and H. Paukkunen, Centrality dependence of inclusive prompt photon production in $\mathrm{d}+\mathrm{Au}$, $\mathrm{Au}+\mathrm{Au}, \mathrm{p}+\mathrm{Pb}$, and $\mathrm{Pb}+\mathrm{Pb}$ collisions, J. High Energy Phys. 05 (2013) 030 . 
[80] M. D. Schwartz, Precision direct photon spectra at high energy and comparison to the $8 \mathrm{TeV}$ ATLAS data, J. High Energy Phys. 09 (2016) 005.

[81] T. Jezo, M. Klasen, and F. König, Prompt photon production and photon-hadron jet correlations with POWHEG, J. High Energy Phys. 11 (2016) 033.

[82] A. V. Lipatov and M. A. Malyshev, Reconsideration of the inclusive prompt photon production at the LHC with $k_{T}$-factorization, Phys. Rev. D 94, 034020 (2016).

[83] A. K. Kohara and C. Marquet, Prompt photon production in double-Pomeron-exchange events at the LHC, Phys. Lett. B 757, 393 (2016).

[84] S. Benic, K. Fukushima, O. Garcia-Montero, and R. Venugopalan, Probing gluon saturation with next-to-leading order photon production at central rapidities in proton-nucleus collisions, J. High Energy Phys. 01 (2017) 115.

[85] F. Siegert, A practical guide to event generation for prompt photon production with Sherpa, J. Phys. G 44, 044007 (2017).

[86] M. Klasen, C. Klein-Bösing, and H. Poppenborg, Prompt photon production and photon-jet correlations at the LHC, J. High Energy Phys. 03 (2018) 081.

[87] M. Goharipour and H. Mehraban, Predictions for the isolated prompt photon production at the LHC at $\sqrt{s}=13 \mathrm{TeV}$, Adv. High Energy Phys. 2017, 3802381 (2017).

[88] J. M. Campbell, R. K. Ellis, and C. Williams, Direct Photon Production at Next-to-Next-to-Leading Order, Phys. Rev. Lett. 118, 222001 (2017).

[89] T. Becher, G. Bell, C. Lorentzen, and S. Marti, Transversemomentum spectra of electroweak bosons near threshold at NNLO, J. High Energy Phys. 02 (2014) 004.

[90] L. Bourhis, M. Fontannaz, and J. P. Guillet, Quark and gluon fragmentation functions into photons, Eur. Phys. J. C 2, 529 (1998).

[91] A. Buckley, J. Ferrando, S. Lloyd, K. Nordström, B. Page, M. Rüfenacht, M. Schönherr, and G. Watt, LHAPDF6: Parton density access in the LHC precision era, Eur. Phys. J. C 75, 132 (2015).

[92] Z. Kunszt and Z. Trocsanyi, QCD corrections to photon production in association with hadrons in $\mathrm{e}^{+} \mathrm{e}^{-}$annihilation, Nucl. Phys. B 394, 139 (1993).

[93] E. W. N. Glover, and A. G. Morgan, Measuring the photon fragmentation function at LEP, Z. Phys. C 62, 311 (1994).

[94] S. Frixione, Isolated photons in perturbative QCD, Phys. Lett. B 429, 369 (1998).

[95] Z. Hall and J. Thaler, Photon isolation and jet substructure, J. High Energy Phys. 09 (2018) 164.

[96] S. Chatrchyan et al. (CMS Collaboration), Measurement of the differential cross section for isolated prompt photon production in $p p$ collisions at $7 \mathrm{TeV}$, Phys. Rev. D 84, 052011 (2011).

[97] G. Aad et al. (ATLAS Collaboration), Measurement of the inclusive isolated prompt photons cross section in $p p$ collisions at $\sqrt{s}=7 \mathrm{TeV}$ with the ATLAS detector using $4.6 \mathrm{fb}^{-1}$, Phys. Rev. D, 89, 052004 (2014).

[98] G. Aad et al. (ATLAS Collaboration), Measurement of the inclusive isolated prompt photon cross section in pp collisions at $\sqrt{s}=8 \mathrm{TeV}$ with the ATLAS detector, J. High Energy Phys. 08 (2016) 005.

[99] M. Aaboud et al. (ATLAS Collaboration), Measurement of the cross section for inclusive isolated-photon production in $p p$ collisions at $\sqrt{s}=13 \mathrm{TeV}$ using the ATLAS detector, Phys. Lett. B 770, 473 (2017).

[100] R. Gauld, J. Rojo, L. Rottoli, and J. Talbert, Charm production in the forward region: constraints on the small- $x$ gluon and backgrounds for neutrino astronomy, J. High Energy Phys. 11 (2015) 009.

[101] R. Gauld and J. Rojo, Precision Determination of the Small-x Gluon from Charm Production at LHCb, Phys. Rev. Lett. 118, 072001 (2017).

[102] R. Aaij et al. (LHCb Collaboration), Observation of $Z$ production in proton-lead collisions at LHCb, J. High Energy Phys. 09 (2014) 030. 